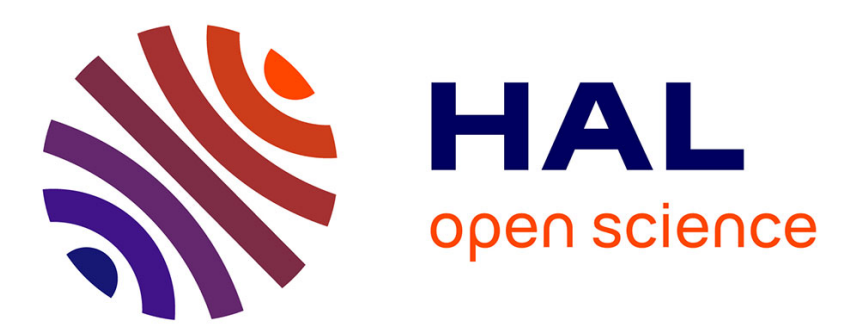

\title{
Vibration sensitive behavior to connecting angle. Case of coupled beams and plates
}

Morvan Ouisse, Jean-Louis Guyader

\section{To cite this version:}

Morvan Ouisse, Jean-Louis Guyader. Vibration sensitive behavior to connecting angle. Case of coupled beams and plates. Journal of Sound and Vibration, 2003, 267 (4), pp.809-850. 10.1016/S0022460X(03)00179-2 . hal-00131979

\section{HAL Id: hal-00131979 \\ https://hal.science/hal-00131979}

Submitted on 13 Mar 2018

HAL is a multi-disciplinary open access archive for the deposit and dissemination of scientific research documents, whether they are published or not. The documents may come from teaching and research institutions in France or abroad, or from public or private research centers.
L'archive ouverte pluridisciplinaire HAL, est destinée au dépôt et à la diffusion de documents scientifiques de niveau recherche, publiés ou non, émanant des établissements d'enseignement et de recherche français ou étrangers, des laboratoires publics ou privés. 
This document is the author's final manuscript of

M. Ouisse, J. L. Guyader, Vibration sensitive behaviour of a connecting angle. Case of coupled beams and plates. Journal of Sound and Vibration, 267(4):809 - 850, 2003. Journal of Sound and Vibration, 260(1):83 - 100, 2003.

This paper has been published by Elsevier and can be found at http://dx.doi.org/10.1016/S0022-460X(03)00179-2 


\section{VIBRATION SENSITIVE BEHAVIOR TO CONNECTING ANGLE. CASE OF COUPLED BEAMS AND PLATES. \\ M. OUISSE AND J.L. GUYADER}

Address:

Institut National des Sciences Appliquées de Lyon

Laboratoire Vibrations Acoustique

25 bis, avenue Jean Capelle

69621 VILLEURBANNE Cedex, France

Short running headline: Vibration sensitive behavior of coupled beams and plates

Number of pages: 77 


\begin{abstract}
:
In this paper one consider semi-infinite $1 \mathrm{D}$ and $2 \mathrm{D}$ coupled structures in order to find conditions under which coupling effects can induce hypersensitive vibrating behavior. The approach is based on classical wave decomposition, and the first point is to show that high sensitivity can exist in such simple systems. Then, for the beams case, it is shown that two critical coupling angles can be defined, and that their values depend only on waves numbers ratio. A similar study is then performed on semi-infinite coupled plates, and existence of critical coupling angle is shown. Its value can be determined using three structural parameters. Results are finally compared to finite coupled structures
\end{abstract}




\section{Introduction}

Uncertainty can be a major questioning source as soon as manufacturing processes are used to build structures. Many reports have been presented [1], [2], in which people show that sensitivity causes can be numerous in complex systems, and are not easily detectable. In our recent works [3], a method that could help one to predict which structural zones are responsible for hypersensitive behavior [4] has been presented. In references [5] and [4], a study concerning coupled plates has been used to show that a small shift of coupling angle could induce very large variations of response, when the nominal coupling angle has a particular value. This value depends on the characteristics of the structure, and has been determined for only a few specific cases. In the present paper we would like to present a much basic study to find values of coupling angles which are in many cases responsible for vibration sensitive behavior. This work is based on a wave approach in semi-infinite coupled structures (coupled beams and coupled plates), in order that the number of structural parameters remains low.

The first part deals with semi-infinite coupled beams. Wave approaches on this kind of structures are well-developed, because of their simplicity, and then use is common in the field of joints between structures studies. Horner and White [6] have presented equations corresponding to coupling effects between bending and in-plane movements due to connecting angle, in terms of transmitted and reflected powers. They shown that for particular joint angle values, transmitted or reflected power could be very sensitive to this angle. Guo [7] has shown results about models for joint behaviors, using masses and stiffnesses, and even if its results are not interpreted in terms of sensitivity, equations that he developed are used here to find conditions under which the coupled structure is sensitive. 
These conditions are derived using a single structural parameter, and linked to results concerning eigenvalues sensitivities of finite coupled beams.

The second part is comparable to the previous one, except that structures are semi-infinite coupled plates. Coupling effects have been described by Langley and Heron [8], while sensitive behavior has been observed for in-plane waves by Park et al. [9] for small values of joint angle. Even if structural parameters are more numerous than coupled beams cases, conditions under which coupled plates can have sensitive behavior are described in this paper and linked to finite plates responses variability.

\section{Study of two coupled semi-infinite beams}

The considered structure is built with two coupled semi-infinite identical beams, presented on figure 1. Constitutive materials are supposed to be homogeneous and isotropic, and Bernoulli's formulation is used to describe the vibrating behavior of the structure. Both bending and longitudinal movements are considered, and are not independent because of coupling angle $\alpha$. Bending movements are belonging to $\left(O, \overrightarrow{x_{1}}, \overrightarrow{y_{1}}\right)$ plane, longitudinal displacements are along $\left(O, \overrightarrow{x_{1}}\right)$ and $\left(O, \overrightarrow{x_{2}}\right)$ axis, while coupling point is $O$.

The notations used for the forced waves decomposition are detailed on appendix A.

\section{bending movement:}

- Beam number 1 (corresponding to $x_{1}<0$ ) is supposed to be the one in which there is an incident bending wave of unit amplitude, traveling toward the coupling point: 


$$
w_{i}\left(x_{1}\right)=e^{-j k x_{1}}
$$

where $j^{2}=1$ and $k=\sqrt{\omega}\left(\frac{\rho S}{E I}\right)^{\frac{1}{4}}$ is the bending wave number of the beam. One should note that this choice of unit amplitude will bring large values of quantities if they are interpreted as MKS values, that is why units corresponding to displacements and powers are not labelled on figures.

Another wave exists in this beam, because of reflection effects on the coupling line. One can distinguish its evanescent and propagative parts:

$$
w_{r}\left(x_{1}\right)=A e^{k x_{1}}+B e^{j k x_{1}}
$$

where $A$ and $B$ are not known.

- Beam number $2\left(x_{2}>0\right)$ movement is imposed by the coupling. Then, the transmitted bending wave can be written as:

$$
w_{t}\left(x_{2}\right)=C e^{-k x_{2}}+D e^{-j k x_{2}}
$$

where $C$ and $D$ are not known.

\section{As far as longitudinal vibrations are concerned:}

- In beam 1 a traveling reflected longitudinal wave is generated by coupling effects:

$$
u_{r}\left(x_{1}\right)=F e^{j \lambda x_{1}}
$$

in which $\mathrm{F}$ is unknown and $\lambda=\omega \sqrt{\frac{\rho}{E}}$ is the longitudinal wave number of the beam.

- In beam 2, a transmitted traveling wave is generated by the coupling: 


$$
u_{t}\left(x_{2}\right)=G e^{-j \lambda x_{2}}
$$

where $\mathrm{G}$ is not known.

\section{$2.1 \quad$ Equations}

Once displacements fields are known, generalized forces can be determined using constitutive laws linking forces and displacement fields, which can be written for bending moment $M$, shear force $T$ and longitudinal force $N$ :

$$
\begin{gathered}
M(x)=E I \frac{d^{2} w}{d x^{2}} \\
T(x)=-E I \frac{d^{3} w}{d x^{3}} \\
N(x)=E S \frac{d u}{d x}
\end{gathered}
$$

These relations are valid if they are used to define cohesion forces corresponding to an external normal oriented toward increasing $x$. It is also possible to define the rotation of the beam cross section:

$$
\Omega(x)=\frac{d w}{d x}
$$

Then, one can determine each of the six unknowns, using continuity equations at coupling point $x=0$ :

- Displacement continuity:

$$
w_{t}(0)=\left(w_{i}(0)+w_{r}(0)\right) \cos \alpha+u_{r}(0) \sin \alpha
$$




$$
u_{t}(0)=-\left(w_{i}(0)+w_{r}(0)\right) \sin \alpha+u_{r}(0) \cos \alpha
$$

- Rotation continuity:

$$
\frac{d w_{t}}{d x_{2}}(0)=\frac{d\left(w_{i}+w_{r}\right)}{d x_{1}}(0)
$$

- Bending moment continuity:

$$
E I \frac{d^{2} w_{t}}{d x_{2}^{2}}(0)=E I \frac{d^{2}\left(w_{i}+w_{r}\right)}{d x_{1}^{2}}(0)
$$

- Shear and normal forces continuity:

$$
\begin{gathered}
-E I \frac{d^{3} w_{t}}{d x_{2}^{3}}(0)=-E I \frac{d^{3}\left(w_{i}+w_{r}\right)}{d x_{1}^{3}}(0) \cos \alpha+E S \frac{d u_{r}}{d x_{1}}(0) \sin \alpha \\
E S \frac{d u_{t}}{d x_{2}}(0)=E I \frac{d^{3}\left(w_{i}+w_{r}\right)}{d x_{1}^{3}}(0) \sin \alpha+E S \frac{d u_{r}}{d x_{1}}(0) \cos \alpha
\end{gathered}
$$

These six equations can be written in the following terms:

$$
\left[\begin{array}{cccccc}
\cos \alpha & \cos \alpha & -1 & -1 & \sin \alpha & 0 \\
\sin \alpha & \sin \alpha & 0 & 0 & -\cos \alpha & 1 \\
1 & j & 1 & j & 0 & 0 \\
1 & -1 & -1 & 1 & 0 & 0 \\
\cos \alpha & -j \cos \alpha & 1 & -j & -j \mu \sin \alpha & 0 \\
\sin \alpha & -j \sin \alpha & 0 & 0 & j \mu \cos \alpha & j \mu
\end{array}\right]\left[\begin{array}{c}
A \\
B \\
C \\
D \\
F \\
G
\end{array}\right]=\left[\begin{array}{c}
-\cos \alpha \\
-\sin \alpha \\
j \\
1 \\
-j \cos \alpha \\
-j \sin \alpha
\end{array}\right]
$$

In which $\alpha$ is the coupling angle and nondimensionnal variable $\mu=\frac{S \lambda}{I k^{3}}$ is the only structural parameter, presented by Park [9] as a nondimensionnal frequency, which can also be expressed as the wave numbers ratio:

$$
\mu=\frac{S \lambda}{I k^{3}}=\left(\frac{E S}{\rho I \omega^{2}}\right)^{\frac{1}{4}}=\frac{k}{\lambda}
$$


This problem has an analytical solution:

$$
\left[\begin{array}{l}
A \\
B \\
C \\
D \\
F \\
G
\end{array}\right]=\left[\begin{array}{c}
\frac{\left(1+j \mu^{2}\right) \sin ^{2} \alpha}{K(\alpha, \mu)} \\
\frac{\left(1-\mu^{2}\right) \sin ^{2} \alpha-j \mu(1-\cos \alpha)^{2}}{K(\alpha, \mu)} \\
\frac{\left(-1+j \mu^{2}\right) \sin ^{2} \alpha-\mu(1-j)(1-\cos \alpha)^{2}}{K(\alpha, \mu)} \\
\frac{j\left(1-\mu^{2}\right) \sin ^{2} \alpha+2 \mu(1+\cos \alpha)^{2}}{K(\alpha, \mu)} \\
\sin \alpha \frac{(\mu(3-j)-3-j) \cos \alpha+(1+j)(\mu+j)}{K(\alpha, \mu)} \\
\sin \alpha \frac{(1+j)(-\mu+j) \cos \alpha-\mu(3-j)-3-j}{K(\alpha, \mu)}
\end{array}\right]
$$

With:

$$
K(\alpha, \mu)=\mu\left(3+2 \cos \alpha+3 \cos ^{2} \alpha\right)+\left(\mu^{2}+j\right)(1-j) \sin ^{2} \alpha
$$

\subsection{Application}

In this section it will be shown that the behavior of the structure can be classified in two categories, highly sensitive to connecting angle or not. The first point is to find an indicator that could be able to determine in which category the considered structure should be classified.

A particular case is considered. Chosen beams have the same characteristics: square sections $(1 \mathrm{~cm}$ by $1 \mathrm{~cm})$, Young's modulus $E=2.1 \times 10^{11} \mathrm{~Pa}$, density $\rho=7800 \mathrm{~kg} / \mathrm{m}^{3}$, and the calculation is performed at frequency $f=100 \mathrm{~Hz}$. Thus the value of the nondimensionnal parameter $\mu$ is 53.5 . Let's point out the evolution of amplitude of propagative wave, which is responsible for far field structural vibration behavior. Figure 2 shows amplitudes of propagative waves versus coupling angle $\alpha$. According to equation 3, the continuous line is obtained by plotting modulus of amplitudes of transmitted propagative bending wave $(\|D\|)$. One should note that this value corresponds to the choice of using 
unit amplitude impinging wave.

On this figure, one can define distinct zones when coupling angle increases: if coupling angle is equal to zero, incident wave is fully transmitted, since the beam is an infinite one without any discontinuity. As soon as the value of $\alpha$ has a non-null value, amplitude of transmitted wave quickly decreases, and for this case, sensitivity of response to coupling angle has a large value on 0-20 degrees range. Then, if coupling angle is still growing, one can define a large angle range on which the sensitivity is very low. Beyond, another high sensitivity behavior zone can be observed, and finally the amplitude of propagative transmitted wave is null when coupling angle reaches its maximum theoretical value 180 degrees.

A similar analysis can be made considering bending reflected wave, and results are comparable, excepted opposite zones corresponding to total transfer or null transfer, which are reversed compared with transmitted waves analysis, like shown on figure 2 .

Calculation also allows one to obtain amplitudes of transmitted and reflected longitudinal waves. These curves present again high sensitivity zones when coupling angle is close to zero or 180 degrees (see fig. 2). However, in a vibro-acoustic process, we are mainly interested by bending movements, which are responsible for sound radiation.

Since the considered problem can be formulated using only one variable which is the wave numbers ratio $\mu$, it could be interesting to obtain an expression of a critical angle versus $\mu$, for which response sensitivity would be maximum. In order to choose the way this critical angle should be defined, let us plot the derivatives of bending propagative waves amplitudes with respect to coupling angle $\alpha$. This is done on figure 3 .

High sensitivity zones can be found again on this figure. The first one is 
centered on an angle of approximately 12 degrees, while the second one is centered on an angle of about 170 degrees. Unfortunately, it is not possible to define a precise value for critical angles that would be a characteristic information for high sensitivity zones, since both transmitted and reflected waves do not reach their maximum sensitivity for the same coupling angle. A way to break this limit, is to consider a power analysis of the structure.

\subsection{Power flow balance}

It is possible to obtain analytical expressions for powers, but for sake of simplicity we won't detail complete expressions here. Considered power flows are defined on the basis that incoming wave $w_{i}$ is an input power of a part of the beam of which normal vector is oriented in $x_{1}<0$ direction, which involves signs inversions in constitutive laws 6 to 8 . This sign convention is also valid for both transmitted and reflected power flows. Let's distinguish the different transmission paths:

Power transmitted by shear force:

$$
\begin{gathered}
P_{T}\left(x_{2}\right)=\frac{1}{2} \operatorname{Re}\left(\overline{\dot{w}_{t}} T_{t}\right) \\
P_{T}\left(x_{2}\right)=\frac{1}{2} E I \omega k^{3}\left(\|D\|^{2}+e^{-k x_{2}} \operatorname{Re}\left(j C \bar{D} e^{j k x_{2}}+\bar{C} D e^{-j k x_{2}}\right)\right)
\end{gathered}
$$

Power transmitted by bending moment:

$$
\begin{gathered}
P_{M}\left(x_{2}\right)=\frac{1}{2} \operatorname{Re}\left(\overline{\dot{\Omega}_{t}} M_{t}\right) \\
P_{M}\left(x_{2}\right)=\frac{1}{2} E I \omega k^{3}\left(\|D\|^{2}-e^{-k x_{2}} R e\left(C \bar{D} e^{j k x_{2}}-j \bar{C} D e^{-j k x_{2}}\right)\right)
\end{gathered}
$$


Thus powers transmitted by shear force and bending moment depend on the point of calculation, the total bending transmitted power (equation 24) is independent of $x$ and remains constant all along beam 2:

$$
P_{\text {bending }}=P_{T}\left(x_{2}\right)+P_{M}\left(x_{2}\right)=E I \omega k^{3}\|D\|^{2}
$$

As far as the total transmitted power is concerned, one must first take into account the third path of transmission, which is due to longitudinal waves:

$$
P_{\text {longi }}=\frac{1}{2} \operatorname{Re}\left(\overline{\dot{u}_{t}} N_{t}\right)=\frac{1}{2} \omega \lambda E S\|G\|^{2}
$$

Then, total transmitted power does not depend on the point chosen for its evaluation:

$$
P_{\text {trans }}=E I \omega k^{3}\|D\|^{2}+\frac{1}{2} E S \omega \lambda\|G\|^{2}
$$

Let's note that this power, which is plotted versus coupling angle on figure 4, allows one to find again which angles ranges are highly sensitive. One should note that the values of power, which are of the order of $10^{7}$, correspond to the use of a unit impinging wave.

As far as incident power is concerned:

$$
\begin{gathered}
P_{i n c}=\frac{1}{2} \operatorname{Re}\left(\overline{\dot{w}_{i}} T_{i}\right)+\frac{1}{2} \operatorname{Re}\left(\overline{\dot{\Omega}_{i}} M_{i}\right) \\
P_{i n c}=\frac{1}{2} \operatorname{Re}\left(\overline{\dot{w}_{i}} E I \frac{d^{3} w_{i}}{d x^{3}}\right)+\frac{1}{2} \operatorname{Re}\left(-\overline{\dot{\Omega}}_{i} E I \frac{d^{2} w_{i}}{d x^{2}}\right) \\
P_{i n c}=E I \omega k^{3}
\end{gathered}
$$


And, finally, reflected power can be written as:

$$
\begin{gathered}
P_{r e f l}=\frac{1}{2} \operatorname{Re}\left(\overline{\dot{w}_{r}} T_{r}\right)+\frac{1}{2} \operatorname{Re}\left(\overline{\dot{\Omega}_{r}} M_{r}\right)+\frac{1}{2} \operatorname{Re}\left(\overline{\dot{u}_{r}} N_{r}\right) \\
P_{r e f l}=-E I \omega k^{3}\|B\|^{2}-\frac{1}{2} E S \omega \lambda\|F\|^{2}
\end{gathered}
$$

The three ways of reflection are illustrated on figure 5, on which one can find sensitive coupling angles ranges.

Thus, power flow balance applied to the considered part of the structure can be easily written since our model does not take into account any losses:

$$
P_{\text {inc }}+P_{\text {refl }}=P_{\text {trans }}
$$

where $P_{\text {inc }}>0 ; P_{\text {refl }}<0 ; P_{\text {trans }}>0$, which is in accordance with propagation direction of each considered wave.

The interest of the power analysis is that transmitted and reflected waves sensitivity to coupling angles are identical, thanks to power balance, since incoming power does not depends on coupling angle value. On figure 6 , power derivative curve is presented, allowing one to define two angles for which the sensitivity is very high. The first angle has a value of 10 degrees, where as the second one is 172 degrees. These results are in accordance with those concerning displacements analysis, and the interest is that one can characterize the structure by two "critical" angles, around which transmitted (or reflected) power sensitivity is large. These critical angles characterize structure sensitivity to coupling angle, which is due to rapid changes of power flow. 


\subsection{Critical angles values}

It has been shown that the formulation could be made using only one variable, which is the wave numbers ratio. Thus it is possible to calculate critical angles versus parameter $\mu$. Figure 7 shows numerical results: critical angles values are plotted versus $\mu$, which belongs to classical structures ranges.

One should note that the small critical angle does not always exist. Indeed, when $\frac{1}{3}<\mu<3$, there is only one maximum on sensitivity curve, which is above 90 degrees. The limit case between existence and non-existence of the first angle is shown on figure 8 .

\subsection{Conclusion on coupled semi-infinite beams}

Finally, it has been shown that for semi-infinite coupled beams, only one structural parameter was enough to characterize the sensitivity of the structure with respect to coupling angle. The existence of critical angle is due to rapid changes of power flow. The parameter used here is the waves numbers ratio. In most of envisaged cases, two critical angles can be defined, around which sensitivity is strong. One of these has a value lower than $45^{\circ}$, while the other is greater than $130^{\circ}$. Critical angles values have been evaluated as functions of waves numbers ratio.

\subsection{Analysis of finite coupled beams}

Previous results have been obtained using power flow analysis of semi infinite coupled beams, so one can wonder if critical angles which have been defined in this way can be linked with results concerning finite structures.

Two finite beams, which are described on figure 9, are coupled with an angle 
$\alpha$, and simply supported on both ends. Notations for this structure are detailed in appendix A. A modal analysis of these coupled beams is presented here.

Motion equations for beams $1\left(x_{1} \in\left[0, L_{1}\right]\right)$ and $2\left(x_{2} \in\left[-L_{2}, 0\right]\right)$ are $(i=1$ and 2):

$$
\left\{\begin{array}{l}
\rho_{i} S_{i} \omega^{2} w_{i}\left(x_{i}\right)-E_{i} I_{i} \frac{d^{4} w_{i}\left(x_{i}\right)}{d x_{i}^{4}}=0 \\
\rho_{i} S_{i} \omega^{2} u_{i}\left(x_{i}\right)+E_{i} S_{i} \frac{d^{2} u_{i}\left(x_{i}\right)}{d x_{i}^{2}}=0
\end{array}\right.
$$

This is a very classical problem, its general solution can be written using this form:

$$
\begin{gathered}
w_{i}\left(x_{i}\right)=\alpha_{i} \cos k_{i} x_{i}+\beta_{i} \sin k_{i} x_{i}+\gamma_{i} \operatorname{ch} k_{i} x_{i}+\delta_{i} \operatorname{sh} k_{i} x_{i} \\
u_{i}\left(x_{i}\right)=\varepsilon_{i} \cos \lambda_{i} x_{i}+\xi_{i} \sin \lambda_{i} x_{i}
\end{gathered}
$$

In which wave numbers are:

$$
\begin{gathered}
k_{i}^{4}=\frac{\rho_{i} S_{i} \omega^{2}}{E_{i} I_{i}} \\
\lambda_{i}^{2}=\omega^{2} \frac{\rho_{i}}{E_{i}}
\end{gathered}
$$

Boundary conditions on points $x_{i}=0$ can be used in order to simplify above equations by canceling $\alpha_{i}, \gamma_{i}$ and $\xi_{i}$ coefficients. Coupling conditions at points $x_{1}=L_{1}$ and $x_{2}=-L_{2}$ are similar to equations 10 to 15 , and allow one to obtain this linear system:

$$
T X=0
$$


In which $X^{T}=\left[\begin{array}{llllll}\beta_{1} & \delta_{1} & \beta_{2} & \delta_{2} & \xi_{1} & \xi_{2}\end{array}\right]$

and:

$T=\left[\begin{array}{cccccc}\sin k_{1} L_{1} & \operatorname{sh} k_{1} L_{1} & \sin _{2} L_{2} \cos \alpha & \operatorname{sh} k_{2} L_{2} \cos \alpha & 0 & -\cos \lambda_{2} L_{2} \sin \alpha \\ 0 & 0 & -\sin k_{2} L_{2} \sin \alpha & -\operatorname{sh} k_{2} L_{2} \sin \alpha & \cos \lambda_{1} L_{1} & -\cos \lambda_{2} L_{2} \cos \alpha \\ \cos k_{1} L_{1} & \operatorname{ch} k_{1} L_{1} & -\frac{k_{2}}{k_{1}} \cos k_{2} L_{2} & -\frac{k_{2}}{k_{1}} \operatorname{ch} k_{2} L_{2} & 0 & 0 \\ -\sin k_{1} L_{1} & \operatorname{sh} k_{1} L_{1} & -\frac{k_{2}^{2}}{k_{1}^{2}} \sin k_{2} L_{2} & \frac{k_{2}^{2}}{k_{1}^{2}} \operatorname{sh} k_{2} L_{2} & 0 & 0 \\ \cos k_{1} L_{1} & -\operatorname{ch} k_{1} L_{1} & -\frac{E_{2} I_{2} k_{2}^{3}}{E_{1} I_{1} k_{1}^{3}} \cos k_{2} L_{2} \cos \alpha & \frac{E_{2} I_{2} k_{2}^{3}}{E_{1} I_{1} k_{1}^{3}} \operatorname{ch} k_{2} L_{2} \cos \alpha & 0 & \frac{E_{2} S_{2} \lambda_{2}}{E_{1} I_{1} k_{1}^{3}} \sin \lambda_{2} L_{2} \sin \alpha \\ 0 & 0 & \frac{E_{2} I_{2} k_{2}^{3}}{E_{1} S_{1} \lambda_{1}} \cos k_{2} L_{2} \sin \alpha & -\frac{E_{2} I_{2} k_{2}^{3}}{E_{1} S_{1} \lambda_{1}} \operatorname{ch} k_{2} L_{2} \sin \alpha & -\sin \lambda_{1} L_{1} & \frac{E_{2} S_{2} \lambda_{2}}{E_{1} S_{1} \lambda_{1}} \sin \lambda_{2} L_{2} \cos \alpha\end{array}\right]$

Eigenvalues of the structure are finally obtained with a numerical resolution of non linear equation $\operatorname{det}(T)=0$.

\subsection{Numerical application}

The chosen characteristics are: $E_{i}=2,1.10^{11} \mathrm{~Pa}, \rho_{i}=7800 \mathrm{~kg} \cdot \mathrm{m}^{-3}, L_{1}=35 \mathrm{~cm}$, $L_{2}=27 \mathrm{~cm}$. Beams rectangular sections are identical $(3 \mathrm{~cm}$ by $1 \mathrm{~cm})$. Then, numerical resolution of $\operatorname{det}(T)=0$ allows one to obtain figure 10 , on which the ten first eigenfrequencies of the structure are plotted versus coupling angle $\alpha$. Some of these frequencies are very sensitive to coupling angle, in particular those corresponding to modes $4,6,8$ and 10 . One can note that these situations are generally local modes of one beam or correspond to in-phase vibrating beams.

\subsection{Relationship between critical angle and eigen frequency sensitivity}

On figure 10, inflexion points are indicated, corresponding to angle for which sensitivity of considered eigenfrequency with respect to coupling angle reaches its 
maximum value. Each of these points can be used in order to define a "critical angle" associated to an eigenfrequency , allowing one to calculate the corresponding wave number ratio using equation 17 . This set of points can be plotted on figure 7, which characterizes critical angles for semi-infinite beams. This is done on figure 11, on which one can observe that critical angles defined using energy considerations of coupled semi-infinite beams are close to the ones defined using sensitivity of eigenfrequencies of coupled finite beams. The only point which is not really close to the original curve is the one associated with the first mode, but as far as this particular mode is concerned, one can observe on figure 10 that the variation of its eigenfrequency is quite slow when coupling angle grows: in this kind of situation, the use of a so defined critical angle has less meaning compared with a more sensitive mode, like the fourth one.

\subsection{Conclusions on coupled beams}

An analysis of coupled beams has been done in order to show that the behavior of such a simple structure could be very sensitive to coupling angle. This phenomena has been described using semi-infinite beams, for which it has been shown that critical coupling angle was defined using only one parameter, which is the wave numbers ratio. This analysis has been done considering power flows, and can be validated considering finite coupled beams, for which it has been shown that eigenfrequencies variations versus coupling angle could linked to results obtained with semi-infinite beams. 


\section{Coupled plates study}

In this section, a similar study is presented, based on semi-infinite coupled identical plates. Hypersensitivity phenomenon have been observed on coupled plates [4], that's why one can wonder if some simple rules like the ones presented above for beams exist for coupled plates.

Notations used for the forced waves decomposition are detailed on appendix B.

\subsection{Bending movement}

One suppose that an incident bending wave of incidence angle $\theta$ is traveling in plate number 1 , like shown on figure 12 . This incident wave is denoted:

$$
w_{i}=e^{-j k_{x} x-j k_{y} y}
$$

in which $k_{x}=k \cos \theta, k_{y}=k \sin \theta$ and bending wave number $k$ satisfies the dispersion equation:

$$
k^{2}=\omega \sqrt{\frac{\rho h}{D}}
$$

This wave is partly reflected on plate 1, while another part is transmitted on plate 2. Connecting angle couples bending and in-plane vibrations because of boundary conditions on joint line. Taking into account spatial coincidence along coupling line and denoting $k_{e}=k \sqrt{1+\cos ^{2} \theta}$ the near-field wave number, reflected bending wave $w_{r}$ and transmitted one $w_{t}$ can be written:

$$
\left\{\begin{array}{c}
w_{r}=e^{-j k_{x} x}\left(A e^{j k_{y} y}+B e^{k_{e} y}\right) \\
w_{t}=e^{-j k_{x} x}\left(H e^{-j k_{y} y}+K e^{-k_{e} y}\right)
\end{array}\right.
$$




\subsection{In-plane vibrations}

Continuity conditions along joint line introduce coupling effects between bending and in-plane vibrations. Details are given in annex $\mathrm{C}$, and complete equations and derivations can be found in reference [9].

One should distinguish two types of in-plane waves, the longitudinal ones (which are parallel to propagation direction), with a wave number $\lambda$, and the in-plane shear ones (which are perpendicular to propagation direction), corresponding to another wave number $\mu$.

The nature of in-plane waves depends on corresponding wave numbers values in comparison with imposed bending one. The most frequent situation is the case numbered 3 in annex $\mathrm{C}$, when $x$ component of imposed bending wave number is greater than in-plane stress wave number: $k_{x}>\mu$

In this situation, all existing in-plane waves are vanishing ones, corresponding to $y$ components of wave numbers:

$$
\left\{\begin{array}{l}
k_{l y}=j \sqrt{-\lambda^{2}+k_{x}^{2}} \\
k_{s y}=j \sqrt{-\mu^{2}+k_{x}^{2}}
\end{array}\right.
$$

Reflected in-plane waves $u_{r}, v_{r}$ and transmitted ones $u_{t}, v_{t}$ can be expressed as follows:

$$
\begin{aligned}
& \left\{\begin{array}{l}
u_{r}=e^{-j k_{x} x}\left(\frac{k_{x}}{k} C e^{-j k_{l y} y}-\frac{k_{s y}}{k} P e^{-j k_{s y} y}\right) \\
v_{r}=e^{-j k_{x} x}\left(\frac{k_{l y}}{k} C e^{-j k_{l y} y}+\frac{k_{x}}{k} P e^{-j k_{s y} y}\right)
\end{array}\right. \\
& \left\{\begin{array}{c}
u_{t}=e^{-j k_{x} x}\left(\frac{k_{x}}{k} F e^{j k_{l y} y}+\frac{k_{s y}}{k} Q e^{j k_{s y} y}\right) \\
v_{t}=e^{-j k_{x} x}\left(-\frac{k_{l y}}{k} F e^{j k_{l y} y}+\frac{k_{x}}{k} Q e^{j k_{s y} y}\right)
\end{array}\right.
\end{aligned}
$$




\subsection{Constitutive laws along $(\mathrm{O}, \mathrm{x})$ axis :}

Constitutive laws can be written along $(O, x)$ axis to obtain expression of generalized forces, rotation and bending moment using displacement fields that will be used for continuity relations expressions:

$$
\left\{\begin{array}{c}
F_{x}=\frac{E h}{2(1+\nu)}\left(\frac{\partial u}{\partial y}+\frac{\partial v}{\partial x}\right) \\
F_{y}=\frac{E h}{\left(1-\nu^{2}\right)}\left(\nu \frac{\partial u}{\partial x}+\frac{\partial v}{\partial y}\right) \\
F_{z}=-D\left(\frac{\partial^{3} w}{\partial y^{3}}+(2-\nu) \frac{\partial^{3} w}{\partial x^{2} \partial y}\right)
\end{array}\right.
$$

$F_{x}, F_{y}$ and $F_{z}$ are line force densities in $x, y$ and $z$ directions.

$$
\left\{\begin{array}{c}
R=\frac{\partial w}{\partial y} \\
M=D\left(\frac{\partial^{2} w}{\partial y^{2}}+\nu \frac{\partial^{2} w}{\partial x^{2}}\right)
\end{array}\right.
$$

$R$ is the rotation angle and $M$ is the bending moment.

\subsection{Continuity relations}

Eight continuity relations can be written on the junction line, in order to identify wave amplitudes. These relations are:

a) Continuity of components of displacement: $u_{r}=u_{t} ; v_{r}-v_{t} \cos \alpha-$ $w_{t} \sin \alpha=0 ; w_{i}+w_{r}+v_{t} \sin \alpha-w_{t} \cos \alpha=0$

b) Continuity of components of force: $F_{x}^{r}=F_{x}^{t} ; F_{y}^{r}-F_{y}^{t} \cos \alpha-F_{z}^{t} \sin \alpha=0$ ; $\quad F_{z}^{i}+F_{z}^{r}+F_{y}^{t} \sin \alpha-F_{z}^{t} \cos \alpha=0$

c) Continuity of rotation: $R_{i}+R_{r}-R_{t}=0$ and

d) Continuity of bending moment: $M_{i}+M_{r}-M_{t}=0$

These equations can be developed from waves in both plates and lead to the 
linear system of waves amplitudes that can be expressed with only four independent structural parameters: Poisson ratio $\nu$, incidence angle of bending wave $\theta$, coupling angle $\alpha$ and a nondimensionnal parameter:

$$
\xi=\rho \frac{h^{2} \omega^{2}}{12 E}
$$

Finally inversion of the system allows one to obtain displacement field on both plates, and lastly to study behavior sensitivity as previously done for beams.

\subsection{Numerical application:}

Considered structure is made of steel plates $\left(E=2.1 \times 10^{11} \mathrm{~Pa} ; \rho=7800 \mathrm{~kg} / \mathrm{m}^{3}\right.$; $h=2 \mathrm{~mm} ; \nu=0,3)$, while frequency chosen for calculation is $500 \mathrm{~Hz}$ and incident angle is $\theta=40^{\circ}$. Thus nondimensionnal parameter is $1.2 \times 10^{-7}$. Let us observe bending response of the structure, which is responsible for sound radiation, on several points of the structure, in order to observe near and far fields. The corresponding curves are plotted using on one hand a point on the coupling line, since on this point evanescent and traveling waves contribute significantly to the displacement, and on the other hand another point will be used, chosen far from the junction in order that only propagative waves effects can be observed.

Figure 13 shows that bending displacement is very sensitive to coupling angle up to 10 degrees. For greater angles, bending response is not sensitive to angle variation. These observations are made for coupling angles belonging to 0-90 degrees range. Beyond, one can observe similar results to those obtained for coupled beams. These cases will be studied in section 3.6.

As far as in-plane movements are concerned, their evolutions are plotted on figure 14, on which one can see that sensitive behavior exists also for lower cou- 
pling angles. But in the present case, these waves are evanescent, and their amplitudes are decreasing fast when observation point moves away from coupling line.

To define connecting angle of maximum sensitivity, a similar remark as the one done for beams can be made: angles for which sensitivity is maximum depends on the considered wave. In order to rid of this difficulty, let's study transmitted and reflected powers. On figure 15, one can observe that power is mainly transmitted by transverse velocity, whatever coupling angle value may be, while the second transmission path is due to rotation velocity. In-plane movement does not carry any power since associated waves are evanescent. High sensitivity values which have been observed for small coupling angles can be found again on powers evolutions, since for flat angle, reflected power is null, and grows up fast with coupling angle. In a complementary way, transmitted power is maximal for flat coupling angle, then decreases until coupling angle is 5.5 degrees, for which power is fully reflected. Beyond, for larger angles, an equilibrium is established, and power variation is very weak when coupling angle grows up.

One should precise that chosen parameters correspond to case number 3 ( $k_{x}>\mu$ ), and for all structures belonging to that case, evolutions of displacements and powers versus coupling angles are similar to those presented above. This behavior is observed for most of structures: indeed, $k_{x}>\mu$ corresponds to:

$$
\frac{1-\nu}{1+\nu} \frac{3 E}{4 \rho \pi^{2}} \cdot \frac{\cos ^{4} \theta}{h^{2} f^{2}}>1
$$

If one consider steel material $\left(E=2,1.10^{11} \mathrm{~Pa}, \rho=7800 \mathrm{~kg} \cdot \mathrm{m}^{-3}, \nu=0,3\right)$ or aluminum one $\left(E=7,2 \cdot 10^{10} \mathrm{~Pa}, \rho=2700 \mathrm{~kg} \cdot \mathrm{m}^{-3}, \nu=0,34\right)$, the previous relation can be written: 


$$
\frac{\cos ^{4} \theta}{h^{2} f^{2}}>10^{-6}
$$

Thus, let's consider the case of many incident waves, coming from each possible incident angles, one can see on equation 50 that the only waves that will induce propagative in-plane waves will have almost normal incidence. If we apply the above equation on the considered case $(h=2 \mathrm{~mm}, f=500 \mathrm{~Hz})$, incidence angle value beyond which in-plane far-field waves will appear is $88.2^{\circ}$. Then, if we consider an almost normal incident angle $\left(89^{\circ}\right)$, transmitted power will be mainly transported by in-plane waves. For all coupling angles with values below $88.2^{\circ}$, transmission will always be done by bending motion.

In order to characterize hypersensitivity, one can evaluate the critical angle when structural parameters are varied. Like for beams, the critical angle is defined by the angle $\alpha$, for which the derivative of transmitted (or reflected) power with respect to coupling angle, reaches its maximum absolute value. This calculation is performed using the three structural parameters $\theta, \xi$ and $\nu$. Chosen ranges are $10^{-13}<\xi<10^{-3}, 0,2<\nu<0,4$ and $0^{\circ}<\theta<90^{\circ}$, in order to represent most of "classical" structures and materials ranges. Results are shown on figure 16 and 17. One can observe that in general critical angles are lower than 10 degrees, only normal incident waves, high frequencies calculations or plates with large thicknesses bring to larger critical angles. In addition, influence of Poisson ratio is very weak. If one try to extrapolate these results to coupled finite plates, one can suppose that incident waves will come from many directions, and that globally there will be a range of angles for which sensitivity is important. For many situations, this will result in one particular angle that will be more sensitive than the others, and this angle is likely to be lower than 10 degrees, which is in 
accordance with observed results [4].

\subsection{Behavior of coupled plates around $\alpha=180^{\circ}$}

A similar study can be performed for angles around 180 degrees, even if results should be interpreted with precautions, since such structures could be impossible to built. Nevertheless, the mathematical model allows one to obtain figure 18, on which transmitted and reflected powers are plotted versus coupling angle. A high sensitivity zone can be observed near 180 degrees, which can be interpreted as the symmetric effect of the one studied in the previous section. A remark can be made about the particular value $\alpha=180^{\circ}$. In this situation, results observed for beams are no longer valid, since power is partly transmitted, because of effects along $x$ line, due to incidence angle $\theta$, which is 40 degrees in the considered case. Thus, $x$ component of incident wave is not blocked by the geometry of the junction and movement is partly transmitted on plate 2 .

This figure should be compared with figure 19, in which incidence angle is close to 90 degrees. In this situation, behavior is close to the one observed for beams: when coupling angle is $180^{\circ}$, transmitted power is close to zero.

The high sensitivity zone can be characterized by angle for which derivative of transmitted (or reflected) power with respect to connecting angle is maximum. This "critical" angle is plotted versus incident angle $\theta$ and non dimensional parameter $\xi$, for $\nu=0.3$ on figure 20 . The gap observed around $\theta=35^{\circ}$ is due to the chosen definition of critical coupling angle, and can be explained using figures 18 and 19. On figure 18, critical angle is localized around 176 degrees, on the left part of the minimum of reflected power, while as far as the picture plotted with $\theta=85^{\circ}$ is concerned, critical angle is about 173 degrees, but it is localized on the right part of the minimum of reflected power. Transition between these two 
situations implies the existence of the gap observed on figure 20 .

\subsection{In-plane incident waves}

In order to obtain a complete description of coupled plates sensitivity phenomenon, one should wonder if previous results are valid for in-plane incident wave. That could be important, since as far as finite coupled plates are concerned, all kinds of exciting waves can exist. Let's first study effects of an incident in-plane longitudinal wave:

$$
\left\{\begin{array}{l}
u_{i}=\frac{k_{x}}{\lambda} e^{-j k_{x} x-j k_{l y} y} \\
v_{i}=\frac{k_{l y}}{\lambda} e^{-j k_{x} x-j k_{l y} y}
\end{array}\right.
$$

This wave is supposed to reach coupling line with an incident angle $\theta$ :

$$
\left\{\begin{array}{l}
k_{x}=\lambda \cos \theta \\
k_{l y}=\lambda \sin \theta
\end{array}\right.
$$

Complete derivation of equations is not presented here, but the principle is exactly the same as in sections 3.1 and 3.2. Corresponding systems are presented in annex E. All in-plane waves are propagative ones, but as far as bending waves are concerned, one should distinguish two cases:

- $k \geq k_{x}$ in which $k$ is the bending wave number: $k^{2}=\omega \sqrt{\frac{\rho h}{D}}$. In that case, propagative bending waves exist.

- $k<k_{x}$ then bending waves are only evanescent ones.

Let's note that the first case can be considered only if:

$$
h^{2} f^{2} \cos ^{4} \theta \leq 9.10^{6}
$$


which means that most of structures do not satisfy this criterion, and that bending reflected and transmitted waves are generally partly far-field ones.

Then, continuity conditions at plates junctions allow one to solve the problem, and to find transmitted and reflected powers expressions associated to incident in-plane longitudinal wave, like shown on figure 21. The main transmission path is in-plane waves. One can observe that sensitive coupling angles exist, even if sensitivity values are lower than in the previous part.

Research of critical angle corresponding to maximum sensitivity allows one to plot figure 22. One can observe that incident angle has a very low influence on critical angle value, except for low-angled waves, for which critical angle is lower than 10 to 20 degrees (as far as "classical" structures are concerned), which is in accordance with results concerning incident bending wave (section 3.5).

A similar analysis can be performed to obtain figure 23 , which shows corresponding results based on incident in-plane shear wave details on annex F):

$$
\left\{\begin{array}{c}
u_{i}=-\frac{k_{s y}}{\mu} e^{-j k_{x} x-j k_{s y} y} \\
v_{i}=\frac{k_{x}}{\mu} e^{-j k_{x} x-j k_{s y} y}
\end{array}\right.
$$

with:

$$
\left\{\begin{array}{l}
k_{x}=\mu \cos \theta \\
k_{s y}=\mu \sin \theta
\end{array}\right.
$$

One can observe that results are close to those noted above, except for very low incident angles. In those cases, there is no maximum on sensitivity curve. This phenomenon is comparable to the one observed for coupled beams (figure $8)$. 


\subsection{Conclusion on semi-infinite coupled plates and exten- sion to finite coupled plates}

As far as results linked to coupling of semi-infinite plates are concerned, there is no configuration guaranteeing the structure not to be hypersensitive for a given excitation. Nevertheless, the general tendency is that structures with coupling angles close to zero or 180 degrees are most likely to be hypersensitive, since a lot of configurations for which critical angle belongs to these ranges exist. In a general case, the three described kinds of waves can exist simultaneously, but since most of the time bending waves have generally more power than in-plane ones, the behavior of the structure will be close to the first described one (incident bending wave).

The interesting point is that most of critical angles are lower than 10 degrees, and it means that for finite structures, on which incident waves are coming from all directions, there should exist a mean angle for which the structure is very sensitive to coupling angle. This mean angle depends on structure characteristics, but it is possible to affirm that in many cases it will be on the 0-10 degrees range. Of course this result is a general trend and particular values of structural parameters or excitation could imply that critical angle is greater than ten degrees.

This can be verified using results obtained by Rébillard et al. [5], concerning the analysis of two finite plates, which are coupled with an angle $\phi$. Dimensions of the structure are given on figure 24. A sensitivity indicator is defined using transfer mobility between two points (A and $\mathrm{B})$. The mobility $Y(A, B, \phi)$ is the normal velocity at point $\mathrm{B}$ to normal force at point $\mathrm{A}$ ratio. Its variation is denoted $\delta Y(A, B, \delta \phi)$ when coupling angle varies. The sensitivity indicator $\alpha(\phi, \delta \phi)$ 
is then defined:

$$
\alpha(\phi, \delta \phi)=\left|\frac{\delta Y(A, B, \delta \phi)}{Y(A, B, \phi)}\right|
$$

Figure 25, reproduced from [5], represents variation of $\alpha$ at $500 \mathrm{~Hz}$ when the value of $\delta \phi$ is one degree. In this case, maximum sensitivity is obtained for a coupling value of 7.5 degrees.

If one try to compare this result to the one obtained for semi-infinite plates, one should consider the non dimensional parameter 48, whose value is in the considered case $\xi=1.23 \times 10^{-7}$. Figure 26 represents a cut of figure 16 for such a value of $\xi$ : it is not easy to infer from it a particular value for critical angle, since it depends on incident angle.

A simplified approach can be performed assuming that there is a direct combination of waves coming directly from A point to the coupling line, resulting in a global critical angle which is the mean of critical angles taken into account:

$$
\alpha_{\text {critmean }}=\frac{1}{\Delta \theta} \int_{\Delta \theta} \alpha_{\text {crit }}(\theta) d \theta
$$

In the considered case:

$$
\alpha_{\text {critmean }}=\frac{1}{41+49,6}\left(\int_{41}^{90} \alpha_{\text {crit }}(\theta) d \theta+\int_{49,6}^{90} \alpha_{\text {crit }}(\theta) d \theta\right)
$$

Indeed, the estimated critical angle for the considered plate has a value of 6.9 degrees, which is close to the real one (7.5 degrees). This allows one to justify the previous analysis performed on semi infinite plates. 


\section{Conclusion}

For both considered structures (semi-infinite coupled beams and plates), a critical angle can be defined, for which sensitivity of transmitted (and reflected) power with respect to coupling angle is maximum. Its existence is related to rapid changes in the transmission paths, which are clearly identified by a power flow analysis. The value of this angle depends on the characteristics of both structure and excitation, but is often smaller than 10 degrees, or close to 180 degrees. As far as the case of beams is concerned, wave numbers ratio is sufficient to know the critical angle value, where as three parameters are necessary in the case of coupled semi-infinite plates. These results can be used in order to understand behaviors of finite structures, in which various kinds of waves exist, with many incidences, and above results indicates that it often results in the existence of a critical angle whose value is lower than ten degrees.

\section{References}

[1] E. Blain, D. Aubry, P. Chove and P. Lardeur 1999 Euromech 405: Numerical Modelling Of Uncertainties, Valenciennes, France, 1-6. Influence of parameters dispersion on the vibrating behaviour of spot welded plates.

[2] R. BERNHARD 1996 Proceedings of Inter-Noise 96, Liverpool, England, 2867-2872. The limits of predictabiliy due to manufacturing and environmentally induced uncertainty.

[3] M. Ouisse and J.-L. Guyader 2001 8th International Congress on Sound and Vibration, Hong-Kong, China. Localization of structural zones producing hypersensitive behavior. 
[4] E. Rebillard and J.L. Guyader 1997 Journal of Sound and Vibration, 205, no. 3, 337-354. Vibrational behaviour of lattices of plates: basic behaviour and hypersensitivity phenomena.

[5] E. Rebillard and J.L. Guyader 1995 Journal of Sound and Vibration, 188, no. 3, 435-454. Vibrational behaviour of a population of coupled plates: hypersensitivity to the connexion angle.

[6] J.L. Horner and R.G. White 1991 Journal of Sound and Vibration, 147, no. 1, 87-103. Prediction of vibrational power transmission through bends and joints in beam-like structures.

[7] Y.P. Guo 1997 Journal of the Acoustical Society of America, 97, no. 1, 289-297. Flexural wave transmission through angled structural joints.

[8] R.S. Langley and K.H. Heron 1990 Journal of Sound and Vibration, 143, no. 2, 241-253. Elastic wave transmission through plate/beam junctions.

[9] D.-H. PARK, S.-Y. Hong, H.-G. KIL, and J.-J. JeOn 2001 Journal of Sound and Vibration, 244, no. 4, 651-668. Power flow models and analysis of inplane waves in finite coupled thin plates. 


\section{Appendices}

\section{A Notations for section 2}

$\left(O, \overrightarrow{x_{1}}\right)$ : neutral axis of beam 1

$\overrightarrow{y_{1}}$ : flexural axis of beam 1

$\left(O, \overrightarrow{x_{2}}\right)$ : neutral axis of beam 2

$\overrightarrow{y_{2}}$ : flexural axis of beam 2

$(O, \vec{z})$ : common normal axis to $\left(O, \overrightarrow{x_{1}}, \overrightarrow{y_{1}}\right)$ and $\left(O, \overrightarrow{x_{2}}, \overrightarrow{y_{2}}\right)$ planes

$\alpha$ : coupling angle

$w_{1}$ : flexural displacement of beam 1

$w_{2}$ : flexural displacement of beam2

$k$ : flexural wave number

$\omega:$ frequency of excitation $(\mathrm{rad} / \mathrm{s})$

$S$ : beams sections

$E$ : Young modulus

$I$ : moment of inertia of the beam

$u_{1}$ : longitudinal displacement of beam 1

$u_{2}$ : longitudinal displacement of beam 2

$\lambda$ : longitudinal wave number

$\rho$ : density

$M$ : bending moment

$T$ : shear force

$N$ : longitudinal force

$\Omega$ : rotation of the beam cross section

$\mu$ : wave numbers ratio 
$f$ : frequency of excitation $(\mathrm{Hz})$

$P_{T}$ : power transmitted by shear force

$P_{M}$ : power transmitted by bending moment

$P_{\text {bending }}:$ power transmitted by bending movement

$P_{\text {longi }}$ : power transmitted by longitudinal movement

$P_{\text {trans }}:$ total transmitted power

$P_{\text {inc }}$ : incident power

$P_{\text {refl }}$ : reflected power

$\alpha_{\text {crit }}:$ critical angle value

$L_{i}$ : length of beam $i$

\section{B Notations for section 3}

$\vec{x}$ : coupling line axis

$\left(\vec{x}, \overrightarrow{y_{0}}\right)$ : first plate plane

$\overrightarrow{z_{0}}$ : normal axis to plate 1

$\left(\vec{x}, \overrightarrow{y_{1}}\right)$ : second plate plane

$\overrightarrow{z_{1}}$ : normal axis to plate 2

$\alpha$ : coupling angle

$\theta$ : incident angle of exciting wave

$u, v$ and $w$ : displacement components

$k$ : flexural wave number

$\omega:$ frequency $(\mathrm{rad} / \mathrm{s})$

$\rho$ : density of material

$E$ : Young modulus of material

$h$ : common thickness of plates 
$D$ : flexural rigidity of plates: $D=\frac{E h^{3}}{12\left(1-\nu^{2}\right)}$

$\nu$ : Poisson ratio of material

$\lambda$ : in-plane longitudinal wave number

$\mu$ : in-plane shear wave number

$\xi$ : nondimensionnal parameter $\xi=\rho \frac{h^{2} \omega^{2}}{12 E}$

\section{In-plane vibrations}

Continuity conditions along joint line introduce coupling effects between bending and in-plane vibrations. In-plane equations have been developed in [9], corresponding to governing equations:

$$
\begin{aligned}
& \frac{\partial^{2} u}{\partial x^{2}}+\frac{1-\nu}{2} \frac{\partial^{2} u}{\partial y^{2}}+\frac{1+\nu}{2} \frac{\partial^{2} v}{\partial x \partial y}=-\frac{1-\nu^{2}}{E} \rho \omega^{2} u \\
& \frac{\partial^{2} v}{\partial x^{2}}+\frac{1-\nu}{2} \frac{\partial^{2} v}{\partial y^{2}}+\frac{1+\nu}{2} \frac{\partial^{2} u}{\partial x \partial y}=-\frac{1-\nu^{2}}{E} \rho \omega^{2} v
\end{aligned}
$$

General solution, using variable separation, can written in the following terms:

$$
\begin{aligned}
& u=u_{l}+u_{s} \\
& v=v_{l}+v_{s}
\end{aligned}
$$

Where one can distinguish two types of in-plane waves, the longitudinal ones (which are parallel to propagation direction)

$$
\begin{aligned}
& u_{l}=k_{l x}\left(A_{l} e^{-j k_{l x} x-j k_{l y} y}-B_{l} e^{j k_{l x} x-j k_{l y} y}+C_{l} e^{-j k_{l x} x+j k_{l y} y}-D_{l} e^{j k_{l x} x+j k_{l y} y}\right) \\
& v_{l}=k_{l y}\left(A_{l} e^{-j k_{l x} x-j k_{l y} y}+B_{l} e^{j k_{l x} x-j k_{l y} y}-C_{l} e^{-j k_{l x} x+j k_{l y} y}-D_{l} e^{j k_{l x} x+j k_{l y} y}\right) \\
& \text { with } k_{l x}^{2}+k_{l y}^{2}=\lambda^{2}=\rho \omega^{2} \frac{1-\nu^{2}}{E}
\end{aligned}
$$

And the in-plane shear ones (which are perpendicular to propagation direction): 


$$
\begin{aligned}
& u_{s}=k_{s y}\left(-A_{s} e^{-j k_{s x} x-j k_{s y} y}-B_{s} e^{j k_{s x} x-j k_{s y} y}+C_{s} e^{-j k_{s x} x+j k_{s y} y}+D_{s} e^{j k_{s x} x+j k_{s y} y}\right) \\
& v_{s}=k_{s x}\left(A_{s} e^{-j k_{s x} x-j k_{s y} y}-B_{s} e^{j k_{s x} x-j k_{s y} y}+C_{s} e^{-j k_{s x} x+j k_{s y} y}-D_{s} e^{j k_{s x} x+j k_{s y} y}\right) \\
& \text { in which } k_{s x}^{2}+k_{s y}^{2}=\mu^{2}=2 \rho \omega^{2} \frac{1+\nu}{E}
\end{aligned}
$$

These expressions can be simplified by taking into account the fact that considered plates are semi-infinite, and that displacement fields should be spatially coincident along $x$ axis with bending fields:

$k_{l x}=k_{x}$ and $k_{s x}=k_{x}$

Then, one should distinguish three cases:

First case, longitudinal wave number value is greater than $x$ component of imposed bending wave number: $\lambda \geq k_{x}$ Let us suppose that considered materials are such as Poisson's ratio $\nu$ is lower than 0.5 , thus in-plane stress $\mu$ wave number is always greater than longitudinal wave number $\lambda$, and for the considered case, it leads to $\mu>k_{x}$ since $\mu=2(1-\nu) \lambda$.

Then, $y$ components of in-plane waves numbers values are fixed and are real and positive.

$$
\begin{aligned}
& k_{l y}=\sqrt{\lambda^{2}-k_{x}^{2}} \\
& k_{s y}=\sqrt{\mu^{2}-k_{x}^{2}}
\end{aligned}
$$

Corresponding waves are propagative ones, and taking into account spatial coincidence, reflected in-plane waves can be expressed:

$$
\begin{aligned}
& u_{r}=e^{-j k_{x} x}\left(\frac{k_{x}}{k} C e^{j k_{l y} y}+\frac{k_{s y}}{k} P e^{j k_{s y} y}\right) \\
& v_{r}=e^{-j k_{x} x}\left(-\frac{k_{l y}}{k} C e^{j k_{l y} y}+\frac{k_{x}}{k} P e^{j k_{s y} y}\right)
\end{aligned}
$$

And as far as transmitted in-plane waves are concerned:

$$
\begin{aligned}
& u_{t}=e^{-j k_{x} x}\left(\frac{k_{x}}{k} F e^{-j k_{l y} y}-\frac{k_{s y}}{k} Q e^{-j k_{s y} y}\right) \\
& v_{t}=e^{-j k_{x} x}\left(\frac{k_{l y}}{k} F e^{-j k_{l y} y}+\frac{k_{x}}{k} Q e^{-j k_{s y} y}\right)
\end{aligned}
$$


Second case, $x$ component of imposed bending wave number is greater than longitudinal wave number and smaller than in-plane stress wave number: $\mu \geq k_{x}>\lambda$ This implies that longitudinal wave is a close-field one, while in-plane stress wave is a propagative one:

$$
\begin{aligned}
& k_{l y}=j \sqrt{-\lambda^{2}+k_{x}^{2}} \\
& k_{s y}=\sqrt{\mu^{2}-k_{x}^{2}}
\end{aligned}
$$

Reflected waves can be expressed like this:

$$
\begin{aligned}
& u_{r}=e^{-j k_{x} x}\left(\frac{k_{x}}{k} C e^{-j k_{l y} y}+\frac{k_{s y}}{k} P e^{j k_{s y} y}\right) \\
& v_{r}=e^{-j k_{x} x}\left(\frac{k_{l y}}{k} C e^{-j k_{l y} y}+\frac{k_{x}}{k} P e^{j k_{s y} y}\right)
\end{aligned}
$$

And as far as transmitted in-plane waves are concerned:

$$
\begin{aligned}
& u_{t}=e^{-j k_{x} x}\left(\frac{k_{x}}{k} F e^{j k_{l y} y}-\frac{k_{s y}}{k} Q e^{-j k_{s y} y}\right) \\
& v_{t}=e^{-j k_{x} x}\left(-\frac{k_{l y}}{k} F e^{j k_{l y} y}+\frac{k_{x}}{k} Q e^{-j k_{s y} y}\right)
\end{aligned}
$$

Third case, $x$ component of imposed bending wave number is greater than in-plane stress wave number: $k_{x}>\mu$ All existing in-plane waves are close-field ones.

$$
\begin{aligned}
& k_{l y}=j \sqrt{-\lambda^{2}+k_{x}^{2}} \\
& k_{s y}=j \sqrt{-\mu^{2}+k_{x}^{2}}
\end{aligned}
$$

Reflected waves can be expressed like this:

$$
\begin{aligned}
& u_{r}=e^{-j k_{x} x}\left(\frac{k_{x}}{k} C e^{-j k_{l y} y}-\frac{k_{s y}}{k} P e^{-j k_{s y} y}\right) \\
& v_{r}=e^{-j k_{x} x}\left(\frac{k_{l y}}{k} C e^{-j k_{l y} y}+\frac{k_{x}}{k} P e^{-j k_{s y} y}\right)
\end{aligned}
$$

And as far as transmitted in-plane waves are concerned:

$$
\begin{aligned}
& u_{t}=e^{-j k_{x} x}\left(\frac{k_{x}}{k} F e^{j k_{l y} y}+\frac{k_{s y}}{k} Q e^{j k_{s y} y}\right) \\
& v_{t}=e^{-j k_{x} x}\left(-\frac{k_{l y}}{k} F e^{j k_{l y} y}+\frac{k_{x}}{k} Q e^{j k_{s y} y}\right)
\end{aligned}
$$




\section{Linear system for incident bending wave}

In this annex linear systems according to the three considered cases are presented.

- Case 1: $\mu>\lambda \geq k_{x}$

- Case 2: $\mu \geq k_{x}>\lambda$

- Case 3: $k_{x}>\mu>\lambda$

Linear system is denoted T.X $=b$, in which $X^{t}=[A B C F H K P Q]$, corresponding to waves amplitudes defined on section 3.1 and 3.2 . $T$ is a 8 by 8 matrix, its expression depends on considered case:

$$
T=\left[\begin{array}{ll}
T_{11} & T_{12} \\
T_{21} & T_{22}
\end{array}\right]
$$

First case:

$$
\begin{gathered}
T_{11}=\left[\begin{array}{cccc}
0 & 0 & \frac{k_{x}}{k} & -\frac{k_{x}}{k} \\
0 & 0 & \frac{k_{l y}}{k} & \frac{k_{l y}}{k} \cos \alpha \\
1 & 1 & 0 & \frac{k_{l y}}{k} \sin \alpha \\
0 & 0 & 2 \frac{k_{x} k_{l y}}{k^{2}} & 2 \frac{k_{x} k_{l y}}{k^{2}}
\end{array}\right] \\
T_{12}=\left[\begin{array}{cccc}
0 & 0 & \frac{k_{s y}}{k} & \frac{k_{s y}}{k} \\
\sin \alpha & \sin \alpha & -\frac{k_{x}}{k} & \frac{k_{x}}{k} \cos \alpha \\
-\cos \alpha & -\cos \alpha & 0 & \frac{k_{x}}{k} \sin \alpha \\
0 & 0 & \frac{k_{s y}^{2}-k_{x}^{2}}{k^{2}} & \frac{k_{x}^{2}-k_{s y}^{2}}{k^{2}}
\end{array}\right]
\end{gathered}
$$


$T_{21}=\left[\begin{array}{cccc}0 & 0 & j \frac{k_{l y}^{2}+\nu k_{x}^{2}}{k^{2}} & -j \frac{k_{l y}^{2}+\nu k_{x}^{2}}{k^{2}} \cos \alpha \\ j \frac{D\left(-1+\nu^{2}\right) k_{y}\left(k_{y}^{2}+(2-\nu) k_{x}^{2}\right)}{E h k} & \frac{D\left(-1+\nu^{2}\right) k_{e}\left(k_{e}^{2}+(2-\nu) k_{x}^{2}\right)}{E h k} & 0 & j \frac{k_{l y}^{2}+\nu k_{x}^{2}}{k^{2}} \sin \alpha \\ j \frac{k_{y}}{k} & \frac{k_{e}}{k} & 0 & 0 \\ \frac{k_{y}^{2}+\nu k_{x}^{2}}{k^{2}} & \frac{-k_{e}^{2}+\nu k_{x}^{2}}{k^{2}} & 0 & 0\end{array}\right]$

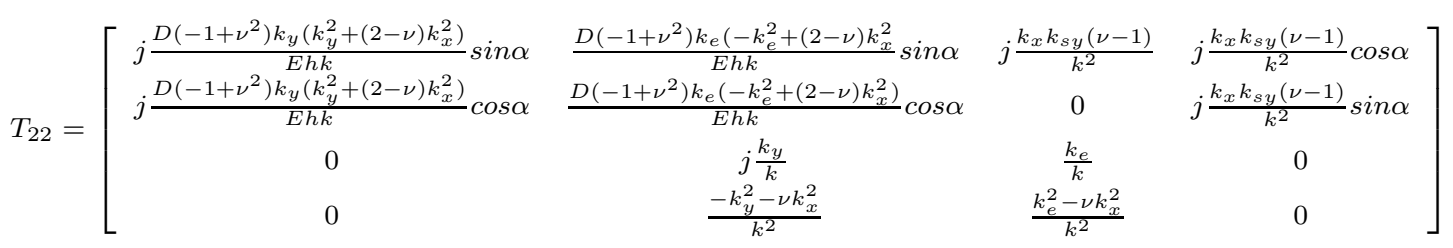

Second case:

$$
\begin{gathered}
T_{11}=\left[\begin{array}{cccc}
0 & 0 & \frac{k_{x}}{k} & -\frac{k_{x}}{k} \\
0 & 0 & -\frac{k_{l y}}{k} & -\frac{k_{l y}}{k} \cos \alpha \\
1 & 1 & 0 & -\frac{k_{l y}}{k} \sin \alpha \\
0 & 0 & -2 \frac{k_{x} k_{l y}}{k^{2}} & -2 \frac{k_{x} k_{l y}}{k^{2}}
\end{array}\right] \\
T_{12}=\left[\begin{array}{cccc}
0 & 0 & \frac{k_{s y}}{k} & \frac{k_{s y}}{k} \\
\sin \alpha & \sin \alpha & -\frac{k_{x}}{k} & \frac{k_{x}}{k} \cos \alpha \\
-\cos \alpha & -\cos \alpha & 0 & \frac{k_{x}}{k} \sin \alpha \\
0 & 0 & \frac{k_{s y}^{2}-k_{x}^{2}}{k^{2}} & \frac{k_{x}^{2}-k_{s y}^{2}}{k^{2}}
\end{array}\right]
\end{gathered}
$$


$T_{21}=\left[\begin{array}{cccc}0 & 0 & j \frac{k_{l y}^{2}+\nu k_{x}^{2}}{k^{2}} & -j \frac{k_{l y}^{2}+\nu k_{x}^{2}}{k^{2}} \cos \alpha \\ j \frac{D\left(-1+\nu^{2}\right) k_{y}\left(k_{y}^{2}+(2-\nu) k_{x}^{2}\right)}{E h k} & \frac{D\left(-1+\nu^{2}\right) k_{e}\left(k_{e}^{2}+(2-\nu) k_{x}^{2}\right)}{E h k} & 0 & j \frac{k_{l y}^{2}+\nu k_{x}^{2}}{k^{2}} \sin \alpha \\ j \frac{k_{y}}{k} & \frac{k_{e}}{k} & 0 & 0 \\ \frac{k_{y}^{2}+\nu k_{x}^{2}}{k^{2}} & \frac{-k_{e}^{2}+\nu k_{x}^{2}}{k^{2}} & 0 & 0\end{array}\right]$

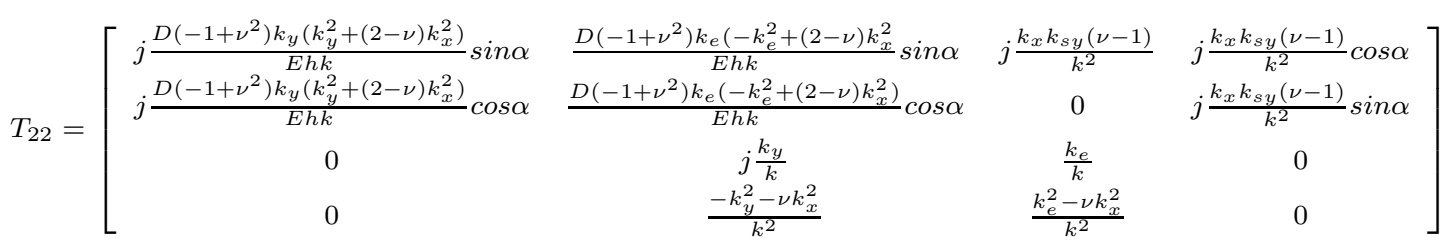

Third case:

$$
\begin{gathered}
T_{11}=\left[\begin{array}{cccc}
0 & 0 & \frac{k_{x}}{k} & -\frac{k_{x}}{k} \\
0 & 0 & -\frac{k_{l y}}{k} & -\frac{k_{l y}}{k} \cos \alpha \\
1 & 1 & 0 & -\frac{k_{l y}}{k} \sin \alpha \\
0 & 0 & -2 \frac{k_{x} k_{l y}}{k^{2}} & -2 \frac{k_{x} k_{l y}}{k^{2}}
\end{array}\right] \\
T_{12}=\left[\begin{array}{cccc}
0 & 0 & -\frac{k_{s y}}{k} & -\frac{k_{s y}}{k} \\
\sin \alpha & \sin \alpha & -\frac{k_{x}}{k} & \frac{k_{x}}{k} \cos \alpha \\
-\cos \alpha & -\cos \alpha & 0 & \frac{k_{x}}{k} \sin \alpha \\
0 & 0 & \frac{k_{s y}^{2}-k_{x}^{2}}{k^{2}} & \frac{k_{x}^{2}-k_{s y}^{2}}{k^{2}}
\end{array}\right]
\end{gathered}
$$


$T_{21}=\left[\begin{array}{cccc}0 & 0 & j \frac{k_{l y}^{2}+\nu k_{x}^{2}}{k^{2}} & -j \frac{k_{l y}^{2}+\nu k_{x}^{2}}{k^{2}} \cos \alpha \\ j \frac{D\left(-1+\nu^{2}\right) k_{y}\left(k_{y}^{2}+(2-\nu) k_{x}^{2}\right)}{E h k} & \frac{D\left(-1+\nu^{2}\right) k_{e}\left(k_{e}^{2}+(2-\nu) k_{x}^{2}\right)}{E h k} & 0 & j \frac{k_{l y}^{2}+\nu k_{x}^{2}}{k^{2}} \sin \alpha \\ j \frac{k_{y}}{k} & \frac{k_{e}}{k} & 0 & 0 \\ \frac{k_{y}^{2}+\nu k_{x}^{2}}{k^{2}} & \frac{-k_{e}^{2}+\nu k_{x}^{2}}{k^{2}} & 0 & 0\end{array}\right]$

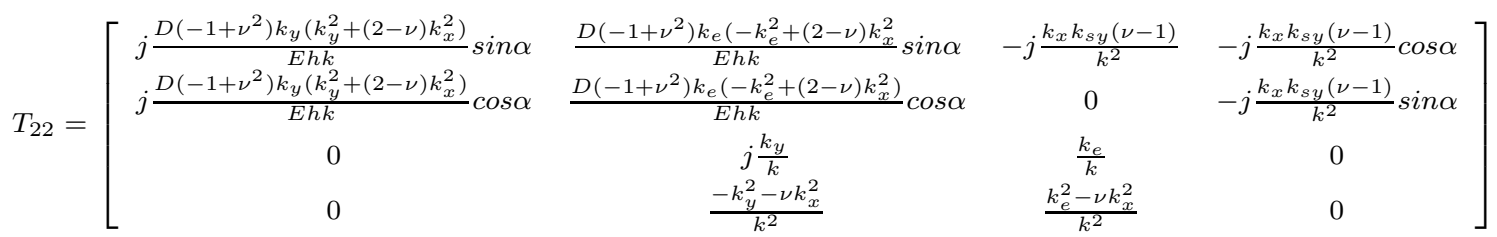

And $b$ is the term corresponding to incident wave:

$$
b=\left[\begin{array}{c}
0 \\
0 \\
-1 \\
0 \\
0 \\
j \frac{D\left(-1+\nu^{2}\right) k_{y}\left(k_{y}^{2}+(2-\nu) k_{x}^{2}\right)}{E h k} \\
j \frac{k_{y}}{k} \\
\frac{-k_{y}^{2}+\nu k_{x}^{2}}{k^{2}}
\end{array}\right]
$$

One can easily show that this linear system can be written using only four parameters:, a non-dimensional parameter $\xi=\rho \frac{h^{2} \omega^{2}}{12 E}$, Poisson ratio $\nu$, connecting angle $\alpha$ and incident angle $\theta$. Expressions of variables used above are:

$$
\begin{aligned}
& \frac{k_{x}}{k}=\cos \theta \\
& \frac{k_{y}}{k}=\sin \theta
\end{aligned}
$$




$$
\begin{aligned}
& \frac{\lambda^{4}}{k^{4}}=\left(1-\nu^{2}\right) \rho \frac{h^{2} \omega^{2}}{12 E}=\left(1-\nu^{2}\right) \xi \\
& \frac{\mu^{4}}{k^{4}}=\frac{4}{(1-\nu)^{2}} \frac{\lambda^{4}}{k^{4}}=4 \frac{1+\nu}{1-\nu} \xi \\
& \frac{k_{e}^{2}}{k^{2}}=1+\cos ^{2} \theta \\
& \frac{k_{l y}^{2}}{k^{2}}=\frac{\lambda^{2}}{k^{2}}-\frac{k_{x}^{2}}{k^{2}}=\sqrt{\left(1-\nu^{2}\right) \xi}-\cos ^{2} \theta \\
& \frac{k_{s y}^{2}}{k^{2}}=\frac{\mu^{2}}{k^{2}}-\frac{k_{x}^{2}}{k^{2}}=2 \sqrt{\frac{1+\nu}{1-\nu} \xi}-\cos ^{2} \theta \\
& \frac{D k^{2}}{E h}=\sqrt{\frac{\xi}{1-\nu^{2}}}
\end{aligned}
$$

\section{E In-plane incident wave}

The considered incident wave is an in-plane longitudinal one of unit amplitude:

$$
\left\{\begin{array}{c}
u_{i}=\frac{k_{x}}{\lambda} e^{-j k_{x} x-j k_{l y} y} \\
v_{i}=\frac{k_{l y}}{\lambda} e^{-j k_{x} x-j k_{l y} y}
\end{array}\right.
$$

Its incidence angle is denoted $\theta$ :

$k_{x}=\lambda \cos \theta$

$k_{l y}=\lambda \sin \theta$

In which $\lambda$ is the longitudinal wave number: $\lambda^{2}=\rho \omega^{2} \frac{1-\nu^{2}}{E}$

Reflected and transmitted in-plane waves are:

$$
\begin{aligned}
& u_{r}=e^{-j k_{x} x}\left(\frac{k_{x}}{k} C e^{j k_{l y} y}+\frac{k_{s y}}{k} P e^{j k_{s y} y}\right) \\
& v_{r}=e^{-j k_{x} x}\left(-\frac{k_{l y}}{k} C e^{j k_{l y} y}+\frac{k_{x}}{k} P e^{j k_{s y} y}\right)
\end{aligned}
$$




$$
\begin{aligned}
& u_{t}=e^{-j k_{x} x}\left(\frac{k_{x}}{k} F e^{-j k_{l y} y}-\frac{k_{s y}}{k} Q e^{-j k_{s y} y}\right) \\
& v_{t}=e^{-j k_{x} x}\left(\frac{k_{l y}}{k} F e^{-j k_{l y} y}+\frac{k_{x}}{k} Q e^{-j k_{s y} y}\right)
\end{aligned}
$$

Considered materials are such as in-plane shear wave number $\mu=2(1-\nu) \lambda$ is greater than $\lambda$, then all in-plane waves are propagative.

As far as bending waves are concerned, one should distinguish two cases:

- First case: $k \geq k_{x}$ in which $k$ is the bending wave number: $k^{2}=\omega \sqrt{\frac{\rho h}{D}}$

Thus $k_{y}=\sqrt{k^{2}-k_{x}^{2}}$ and $k_{e}=\sqrt{k+k_{x}^{2}}$ correspond to traveling and near field parts of reflected and transmitted bending waves:

$$
\begin{gathered}
w_{r}=e^{-j k_{x} x}\left(A e^{j k_{y} y}+B e^{k_{e} y}\right) \\
w_{t}=e^{-j k_{x} x}\left(H e^{-j k_{y} y}+K e^{-k_{e} y}\right)
\end{gathered}
$$

Linear system obtained using continuity relations along coupling line is denoted $T . X=b$, in which $X^{t}=[A B C F H K P Q]$, corresponding to waves amplitudes defined above. $T$ is a 8 by 8 matrix:

$$
T=\left[\begin{array}{ll}
T_{11} & T_{12} \\
T_{21} & T_{22}
\end{array}\right]
$$




$$
\begin{aligned}
& T_{11}=\left[\begin{array}{cccc}
0 & 0 & \frac{k_{x}}{\lambda} & -\frac{k_{x}}{\lambda} \\
0 & 0 & -\frac{k_{l y}}{\lambda} & -\frac{k_{l y}}{\lambda} \cos \alpha \\
1 & 1 & 0 & \frac{k_{l y}}{\lambda} \sin \alpha \\
0 & 0 & 2 \frac{k_{x} k_{l y}}{\lambda k} & 2 \frac{k_{x} k_{l y}}{\lambda k}
\end{array}\right] \\
& T_{12}=\left[\begin{array}{cccc}
0 & 0 & \frac{k_{s y}}{\lambda} & \frac{k_{s y}}{\lambda} \\
-\sin \alpha & -\sin \alpha & \frac{k_{x}}{\lambda} & -\frac{k_{x}}{\lambda} \cos \alpha \\
-\cos \alpha & -\cos \alpha & 0 & \frac{k_{x}}{\lambda} \sin \alpha \\
0 & 0 & \frac{k_{s y}^{2}-k_{x}^{2}}{k \lambda} & \frac{k_{x}^{2}-k_{s y}^{2}}{k \lambda}
\end{array}\right] \\
& T_{21}=\left[\begin{array}{cccc}
0 & 0 & j \frac{k_{l y}^{2}+\nu k_{x}^{2}}{\lambda k} & -j \frac{k_{l y}^{2}+\nu k_{x}^{2}}{\lambda k} \cos \alpha \\
j \frac{D k_{y}\left(k_{y}^{2}+(2-\nu) k_{x}^{2}\right)}{E h k} & \frac{D k_{e}\left(-k_{e}^{2}+(2-\nu) k_{x}^{2}\right)}{E h k} & 0 & j \frac{k_{l y}^{2}+\nu k_{x}^{2}}{\lambda k\left(-1+\nu^{2}\right)} \sin \alpha \\
j \frac{k_{y}}{k} & \frac{k_{e}}{k} & 0 & 0 \\
k_{y}^{2}+\nu k_{x}^{2} & -k_{e}^{2}+\nu k_{x}^{2} & 0 & 0
\end{array}\right] \\
& T_{22}=\left[\begin{array}{cccc}
j \frac{D\left(-1+\nu^{2}\right) k_{y}\left(k_{y}^{2}+(2-\nu) k_{x}^{2}\right)}{E h k} \sin \alpha & \frac{D\left(-1+\nu^{2}\right) k_{e}\left(-k_{e}^{2}+(2-\nu) k_{x}^{2}\right)}{E h k} \sin \alpha & j \frac{k_{x} k_{s y}(\nu-1)}{k \lambda} & j \frac{k_{x} k_{s y}(\nu-1)}{k \lambda} \cos \alpha \\
j \frac{D k_{y}\left(k_{y}^{2}+(2-\nu) k_{x}^{2}\right)}{E h k} \cos \alpha & \frac{D k_{e}\left(-k_{e}^{2}+(2-\nu) k_{x}^{2}\right)}{E h k} \cos \alpha & 0 & -j \frac{k_{x} k_{s y}}{k \lambda(1+\nu)} \sin \alpha \\
j \frac{k_{y}}{k} & \frac{k_{e}}{k} & 0 & 0 \\
-k_{y}^{2}-\nu k_{x}^{2} & k_{e}^{2}-\nu k_{x}^{2} & 0 & 0
\end{array}\right]
\end{aligned}
$$

and the vector $b$ is: 


$$
b=\left[\begin{array}{c}
-\frac{k_{x}}{\lambda} \\
-\frac{k_{y}}{\lambda} \\
0 \\
2 \frac{k_{x} k_{l y}}{\lambda k} \\
-j \frac{k_{l y}^{2}+\nu k_{x}^{2}}{k \lambda} \\
0 \\
0 \\
0
\end{array}\right]
$$

- Second case: $k<k_{x}$

All bending waves are near field ones, with wave numbers $k_{y}=\sqrt{k_{x}^{2}-k^{2}}$ and $k_{e}=\sqrt{k+k_{x}^{2}}$ :

$$
\begin{gathered}
w_{r}=e^{-j k_{x} x}\left(A e^{k_{y} y}+B e^{k_{e} y}\right) \\
w_{t}=e^{-j k_{x} x}\left(H e^{-k_{y} y}+K e^{-k_{e} y}\right)
\end{gathered}
$$

Linear system obtained using continuity relations along coupling line is denoted T.X $=b$, in which $X^{T}=[A B C F H K P Q]$, corresponding to waves amplitudes defined above. $T$ is a 8 by 8 matrix:

$$
T=\left[\begin{array}{ll}
T_{11} & T_{12} \\
T_{21} & T_{22}
\end{array}\right]
$$

Components of $T_{11}$ and $T_{12}$ are similar to those given in case 1. 


$$
T_{21}=\left[\begin{array}{cccc}
0 & 0 & j \frac{k_{l y}^{2}+\nu k_{x}^{2}}{\lambda k} & -j \frac{k_{l y}^{2}+\nu k_{x}^{2}}{\lambda k} \cos \alpha \\
\frac{D k_{y}\left(-k_{y}^{2}+(2-\nu) k_{x}^{2}\right)}{E h k} & \frac{D k_{e}\left(-k_{e}^{2}+(2-\nu) k_{x}^{2}\right)}{E h k} & 0 & j \frac{k_{l y}^{2}+\nu k_{x}^{2}}{\lambda k\left(-1+\nu^{2}\right)} \sin \alpha \\
\frac{k_{y}}{k} & \frac{k_{e}}{k} & 0 & 0 \\
-k_{y}^{2}+\nu k_{x}^{2} & -k_{e}^{2}+\nu k_{x}^{2} & 0 & 0
\end{array}\right]
$$

$T_{22}=\left[\begin{array}{cccc}\frac{D\left(-1+\nu^{2}\right) k_{y}\left(-k_{y}^{2}+(2-\nu) k_{x}^{2}\right)}{E h k} \sin \alpha & \frac{D\left(-1+\nu^{2}\right) k_{e}\left(-k_{e}^{2}+(2-\nu) k_{x}^{2}\right)}{E h k} \sin \alpha & j \frac{k_{x} k_{s y}(\nu-1)}{k \lambda} & j \frac{k_{x} k_{s y}(\nu-1)}{k \lambda} \cos \alpha \\ \frac{D k_{y}\left(-k_{y}^{2}+(2-\nu) k_{x}^{2}\right)}{E h k} \cos \alpha & \frac{D k_{e}\left(-k_{e}^{2}+(2-\nu) k_{x}^{2}\right)}{E h k} \cos \alpha & 0 & -j \frac{k_{x} k_{s y}}{k \lambda(1+\nu)} \sin \alpha \\ \frac{k_{y}}{k} & \frac{k_{e}}{k} & 0 & 0 \\ k_{y}^{2}-\nu k_{x}^{2} & k_{e}^{2}-\nu k_{x}^{2} & 0 & 0\end{array}\right]$

\section{F In-plane shear incident wave}

The imposed wave is a traveling in-plane shear one:

$$
\left\{\begin{array}{c}
u_{i}=-\frac{k_{s y}}{\mu} e^{-j k_{x} x-j k_{s y} y} \\
v_{i}=\frac{k_{x}}{\mu} e^{-j k_{x} x-j k_{s y} y}
\end{array}\right.
$$

Its incidence is denoted $\theta$ :

$k_{x}=\mu \cos \theta$

$k_{s y}=\mu \sin \theta$

In which $\mu^{2}=2 \rho \omega^{2} \frac{1+\nu}{E} ; k^{2}=\omega \sqrt{\frac{\rho h}{D}}$ and $k_{e}=\sqrt{k+k_{x}^{2}}$

3 cases can be distinguished:

- First case: $k>\lambda \geq k_{x}$

Then $k_{y}=\sqrt{k^{2}-k_{x}^{2}}$ and $k_{l y}=\sqrt{\lambda^{2}-k_{x}^{2}}$ :

Reflected waves are:

$$
w_{r}=e^{-j k_{x} x}\left(A e^{j k_{y} y}+B e^{k_{e} y}\right)
$$




$$
\begin{aligned}
& u_{r}=e^{-j k_{x} x}\left(\frac{k_{x}}{k} C e^{j k_{l y} y}+\frac{k_{s y}}{k} P e^{j k_{s y} y}\right) \\
& v_{r}=e^{-j k_{x} x}\left(-\frac{k_{l y}}{k} C e^{j k_{l y} y}+\frac{k_{x}}{k} P e^{j k_{s y} y}\right)
\end{aligned}
$$

Transmitted waves are:

$$
\begin{aligned}
& w_{t}=e^{-j k_{x} x}\left(H e^{-j k_{y} y}+K e^{-k_{e} y}\right) \\
& u_{t}=e^{-j k_{x} x}\left(\frac{k_{x}}{k} F e^{-j k_{l y} y}-\frac{k_{s y}}{k} Q e^{-j k_{s y} y}\right) \\
& v_{t}=e^{-j k_{x} x}\left(\frac{k_{l y}}{k} F e^{-j k_{l y} y}+\frac{k_{x}}{k} Q e^{-j k_{s y} y}\right)
\end{aligned}
$$

All existing waves are traveling ones.

Linear system obtained using continuity relations along coupling line is denoted T.X $=b$, in which $X^{t}=[A B C F H K P Q]$, corresponding to waves amplitudes defined above. $T$ is a 8 by 8 matrix:

$$
\begin{aligned}
& T=\left[\begin{array}{ll}
T_{11} & T_{12} \\
T_{21} & T_{22}
\end{array}\right] \\
& T_{11}=\left[\begin{array}{cccc}
0 & 0 & \frac{k_{x}}{k} & -\frac{k_{x}}{k} \\
0 & 0 & -\frac{k_{l y}}{k} & -\frac{k_{l y}}{k} \cos \alpha \\
1 & 1 & 0 & \frac{k_{l y}}{k} \sin \alpha \\
0 & 0 & 2 \frac{k_{x} k_{l y}}{k^{2}} & 2 \frac{k_{x} k_{l y}}{k^{2}}
\end{array}\right] \\
& T_{12}=\left[\begin{array}{cccc}
0 & 0 & \frac{k_{s y}}{k} & \frac{k_{s y}}{k} \\
-\sin \alpha & -\sin \alpha & \frac{k_{x}}{k} & -\frac{k_{x}}{k} \cos \alpha \\
-\cos \alpha & -\cos \alpha & 0 & \frac{k_{x}}{k} \sin \alpha \\
0 & 0 & \frac{k_{s y}^{2}-k_{x}^{2}}{k^{2}} & \frac{k_{x}^{2}-k_{s y}^{2}}{k^{2}}
\end{array}\right]
\end{aligned}
$$




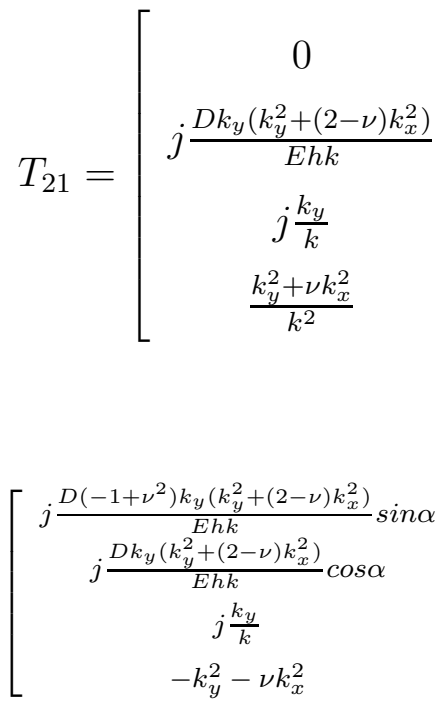

$$
\begin{aligned}
& \left.\begin{array}{ccc}
\frac{D\left(-1+\nu^{2}\right) k_{e}\left(-k_{e}^{2}+(2-\nu) k_{x}^{2}\right.}{E h k} \sin \alpha & j \frac{k_{x} k_{s y}(\nu-1)}{k^{2}} & j \frac{k_{x} k_{s y}(\nu-1)}{k^{2}} \cos \alpha \\
\frac{D k_{e}\left(-k_{e}^{2}+(2-\nu) k_{x}^{2}\right)}{E h k} \cos \alpha & 0 & -j \frac{k_{x} k_{s y}}{(1+\nu) k^{2}} \sin \alpha \\
\frac{k_{e}}{k} & 0 & 0 \\
k_{e}^{2}-\nu k_{x}^{2} & 0 & 0
\end{array}\right] \\
& b=\left[\begin{array}{c}
\frac{k_{s y}}{\mu} \\
-\frac{k_{x}}{\mu} \\
0 \\
\frac{k_{x}^{2}-k_{s y}^{2}}{\mu k} \\
j \frac{k_{s y} k_{x}(\nu-1)}{k \mu} \\
0 \\
0 \\
0
\end{array}\right]
\end{aligned}
$$

- Second case: $k \geq k_{x}>\lambda$

Then $k_{y}=\sqrt{k^{2}-k_{x}^{2}}$ and $k_{l y}=j \sqrt{k_{x}^{2}-\lambda^{2}}$ :

Reflected waves are:

$$
\begin{aligned}
& w_{r}=e^{-j k_{x} x}\left(A e^{j k_{y} y}+B e^{k_{e} y}\right) \\
& u_{r}=e^{-j k_{x} x}\left(\frac{k_{x}}{k} C e^{-j k_{l y} y}+\frac{k_{s y}}{k} P e^{j k_{s y} y}\right)
\end{aligned}
$$


$v_{r}=e^{-j k_{x} x}\left(\frac{k_{l y}}{k} C e^{-j k_{l y} y}+\frac{k_{x}}{k} P e^{j k_{s y} y}\right)$

Transmitted waves are:

$$
\begin{aligned}
& w_{t}=e^{-j k_{x} x}\left(H e^{-j k_{y} y}+K e^{-k_{e} y}\right) \\
& u_{t}=e^{-j k_{x} x}\left(\frac{k_{x}}{k} F e^{j k_{l y} y}-\frac{k_{s y}}{k} Q e^{-j k_{s y} y}\right) \\
& v_{t}=e^{-j k_{x} x}\left(-\frac{k_{l y}}{k} F e^{j k_{l y} y}+\frac{k_{x}}{k} Q e^{-j k_{s y} y}\right)
\end{aligned}
$$

All waves are traveling ones, except in-plane longitudinal ones.

Linear system obtained using continuity relations along coupling line is denoted T.X $=b$, in which $X^{t}=[A B C F H K P Q]$, corresponding to waves amplitudes defined above. $T$ is a 8 by 8 matrix:

$$
\begin{aligned}
& T=\left[\begin{array}{ll}
T_{11} & T_{12} \\
T_{21} & T_{22}
\end{array}\right] \\
& T_{11}=\left[\begin{array}{cccc}
0 & 0 & \frac{k_{x}}{k} & -\frac{k_{x}}{k} \\
0 & 0 & \frac{k_{l y}}{k} & \frac{k_{l y}}{k} \cos \alpha \\
1 & 1 & 0 & -\frac{k_{l y}}{k} \sin \alpha \\
0 & 0 & -2 \frac{k_{x} k_{l y}}{k^{2}} & -2 \frac{k_{x} k_{l y}}{k^{2}}
\end{array}\right] \\
& T_{12}=\left[\begin{array}{cccc}
0 & 0 & \frac{k_{s y}}{k} & \frac{k_{s y}}{k} \\
-\sin \alpha & -\sin \alpha & \frac{k_{x}}{k} & -\frac{k_{x}}{k} \cos \alpha \\
-\cos \alpha & -\cos \alpha & 0 & \frac{k_{x}}{k} \sin \alpha \\
0 & 0 & \frac{k_{s y}^{2}-k_{x}^{2}}{k^{2}} & \frac{k_{x}^{2}-k_{s y}^{2}}{k^{2}}
\end{array}\right]
\end{aligned}
$$




$$
T_{21}=\left[\begin{array}{cccc}
0 & 0 & j \frac{k_{l y}^{2}+\nu k_{x}^{2}}{k^{2}} & -j \frac{k_{l y}^{2}+\nu k_{x}^{2}}{k^{2}} \cos \alpha \\
j \frac{D k_{y}\left(k_{y}^{2}+(2-\nu) k_{x}^{2}\right)}{E h k} & \frac{D k_{e}\left(-k_{e}^{2}+(2-\nu) k_{x}^{2}\right)}{E h k} & 0 & j \frac{k_{l y}^{2}+\nu k_{x}^{2}}{\left(-1+\nu^{2}\right)} \sin \alpha \\
j \frac{k_{y}}{k} & \frac{k_{e}}{k} & 0 & 0 \\
\frac{k_{y}^{2}+\nu k_{x}^{2}}{k^{2}} & \frac{-k_{e}^{2}+\nu k_{x}^{2}}{k^{2}} & 0 & 0
\end{array}\right]
$$

$T_{22}=\left[\begin{array}{cccc}j \frac{D\left(-1+\nu^{2}\right) k_{y}\left(k_{y}^{2}+(2-\nu) k_{x}^{2}\right)}{E h k} \sin \alpha & \frac{D\left(-1+\nu^{2}\right) k_{e}\left(-k_{e}^{2}+(2-\nu) k_{x}^{2}\right.}{E h k} \sin \alpha & j \frac{k_{x} k_{s y}(\nu-1)}{k^{2}} & j \frac{k_{x} k_{s y}(\nu-1)}{k^{2}} \cos \alpha \\ j \frac{D k_{y}\left(k_{y}^{2}+(2-\nu) k_{x}^{2}\right)}{E h k} \cos \alpha & \frac{D k_{e}\left(-k_{e}^{2}+(2-\nu) k_{x}^{2}\right)}{E h k} \cos \alpha & 0 & -j \frac{k_{x} k_{s y}}{(1+\nu) k^{2}} \sin \alpha \\ j \frac{k_{y}}{k} & \frac{k_{e}}{k} & 0 & 0 \\ -k_{y}^{2}-\nu k_{x}^{2} & k_{e}^{2}-\nu k_{x}^{2} & 0 & 0\end{array}\right]$

- Third case: $k_{x}>k>\lambda$

Then $k_{y}=\sqrt{k_{x}^{2}-k^{2}}$ and $k_{l y}=j \sqrt{k_{x}^{2}-\lambda^{2}}$ :

Reflected waves are:

$$
\begin{aligned}
& w_{r}=e^{-j k_{x} x}\left(A e^{k_{y} y}+B e^{k_{e} y}\right) \\
& u_{r}=e^{-j k_{x} x}\left(\frac{k_{x}}{k} C e^{-j k_{l y} y}+\frac{k_{s y}}{k} P e^{j k_{s y} y}\right) \\
& v_{r}=e^{-j k_{x} x}\left(\frac{k_{l y}}{k} C e^{-j k_{l y} y}+\frac{k_{x}}{k} P e^{j k_{s y} y}\right)
\end{aligned}
$$

Transmitted waves are:

$$
\begin{aligned}
& w_{t}=e^{-j k_{x} x}\left(H e^{-k_{y} y}+K e^{-k_{e} y}\right) \\
& u_{t}=e^{-j k_{x} x}\left(\frac{k_{x}}{k} F e^{j k_{l y} y}-\frac{k_{s y}}{k} Q e^{-j k_{s y} y}\right) \\
& v_{t}=e^{-j k_{x} x}\left(-\frac{k_{l y}}{k} F e^{j k_{l y} y}+\frac{k_{x}}{k} Q e^{-j k_{s y} y}\right)
\end{aligned}
$$

All waves are near-field ones, except in-plane shear ones.

Linear system obtained using continuity relations along coupling line is denoted $T . X=b$, in which $X^{t}=[A B C F H K P Q]$, corresponding to waves amplitudes defined above. $T$ is a 8 by 8 matrix: 


$$
\begin{aligned}
& T=\left[\begin{array}{ll}
T_{11} & T_{12} \\
T_{21} & T_{22}
\end{array}\right] \\
& T_{11}=\left[\begin{array}{cccc}
0 & 0 & \frac{k_{x}}{k} & -\frac{k_{x}}{k} \\
0 & 0 & \frac{k_{l y}}{k} & \frac{k_{l y}}{k} \cos \alpha \\
1 & 1 & 0 & -\frac{k_{l y}}{k} \sin \alpha \\
0 & 0 & -2 \frac{k_{x} k_{l y}}{k^{2}} & -2 \frac{k_{x} k_{l y}}{k^{2}}
\end{array}\right] \\
& T_{12}=\left[\begin{array}{cccc}
0 & 0 & \frac{k_{s y}}{k} & \frac{k_{s y}}{k} \\
-\sin \alpha & -\sin \alpha & \frac{k_{x}}{k} & -\frac{k_{x}}{k} \cos \alpha \\
-\cos \alpha & -\cos \alpha & 0 & \frac{k_{x}}{k} \sin \alpha \\
0 & 0 & \frac{k_{s y}^{2}-k_{x}^{2}}{k^{2}} & \frac{k_{x}^{2}-k_{s y}^{2}}{k^{2}}
\end{array}\right] \\
& T_{21}=\left[\begin{array}{cccc}
0 & 0 & j \frac{k_{l y}^{2}+\nu k_{x}^{2}}{k^{2}} & -j \frac{k_{l y}^{2}+\nu k_{x}^{2}}{k^{2}} \cos \alpha \\
\frac{D k_{y}\left(-k_{y}^{2}+(2-\nu) k_{x}^{2}\right)}{E h k} & \frac{D k_{e}\left(-k_{e}^{2}+(2-\nu) k_{x}^{2}\right)}{E h k} & 0 & j \frac{k_{l y}^{2}+\nu k_{x}^{2}}{\left(-1+\nu^{2}\right)} \sin \alpha \\
\frac{k_{y}}{k} & \frac{k_{e}}{k} & 0 & 0 \\
\frac{-k_{y}^{2}+\nu k_{x}^{2}}{k^{2}} & \frac{-k_{e}^{2}+\nu k_{x}^{2}}{k^{2}} & 0 & 0
\end{array}\right] \\
& T_{22}=\left[\begin{array}{cccc}
\frac{D\left(-1+\nu^{2}\right) k_{y}\left(-k_{y}^{2}+(2-\nu) k_{x}^{2}\right)}{E k h} \sin \alpha & \frac{D\left(-1+\nu^{2}\right) k_{e}\left(-k_{e}^{2}+(2-\nu) k_{x}^{2}\right.}{E h k} \sin \alpha & j \frac{k_{x} k_{s y}(\nu-1)}{k^{2}} & j \frac{k_{x} k_{s y}(\nu-1)}{k^{2}} \cos \alpha \\
\frac{D k_{y}\left(-k_{y}^{2}+(2-\nu) k_{x}^{2}\right)}{E k h} \cos \alpha & \frac{D k_{e}\left(-k_{e}^{2}+(2-\nu) k_{x}^{2}\right)}{E h k} \cos \alpha & 0 & -j \frac{k_{x} k_{s y}}{(1+\nu) k^{2}} \sin \alpha \\
\frac{k_{y}}{k} & \frac{k_{e}}{k} & 0 & 0 \\
k_{y}^{2}-\nu k_{x}^{2} & k_{e}^{2}-\nu k_{x}^{2} & 0 & 0
\end{array}\right]
\end{aligned}
$$




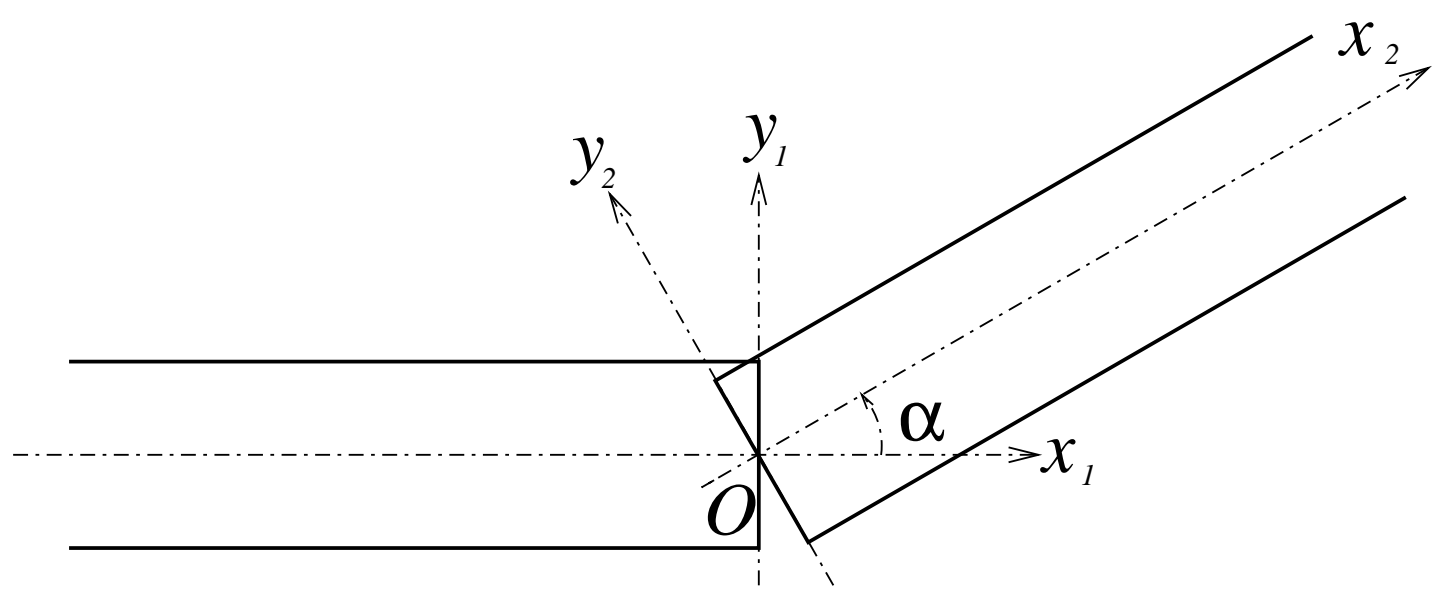

Figure 1: Two coupled semi-infinite beams 


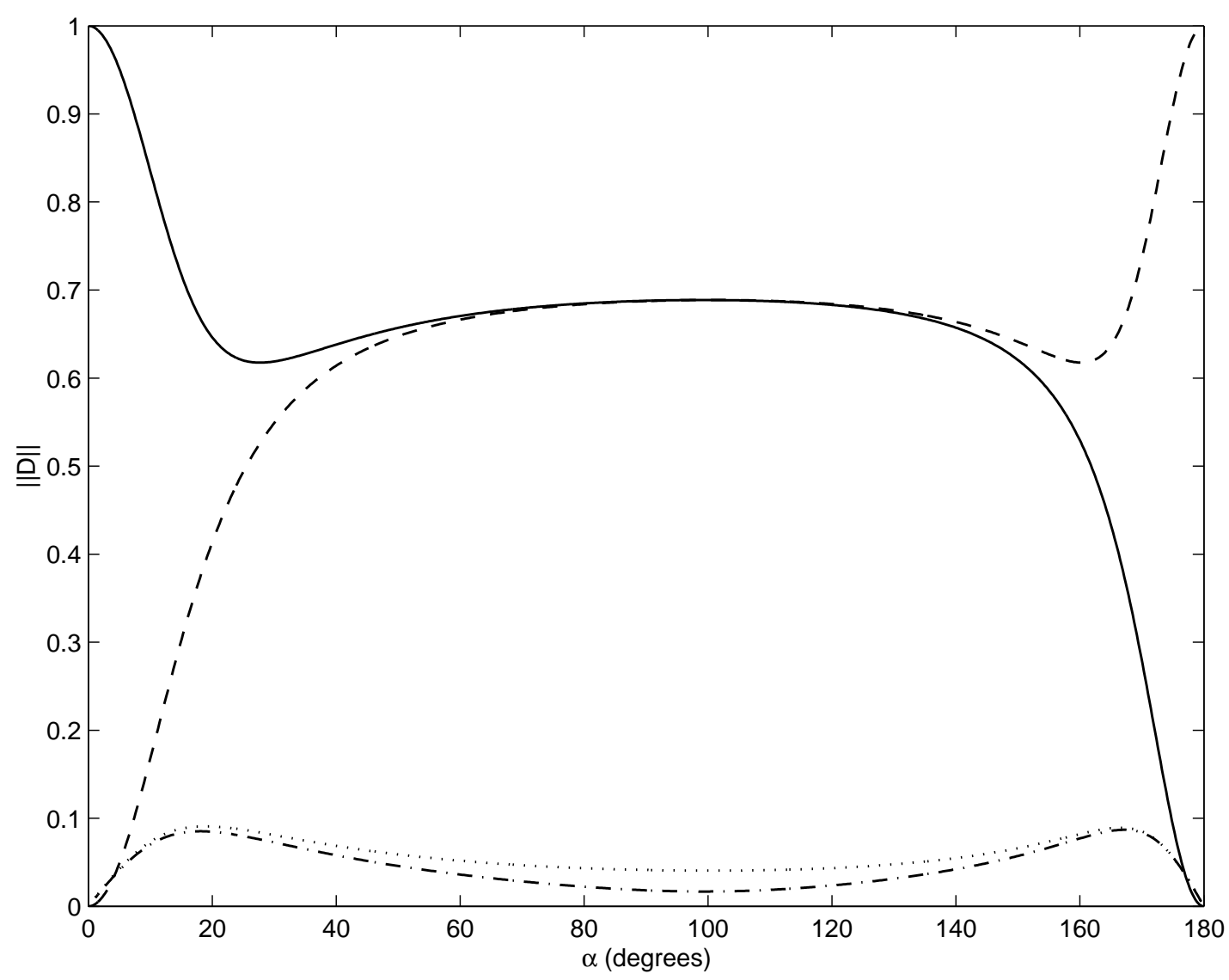

Figure 2: Modulus of amplitudes of propagative waves versus coupling angle. -Bending transmitted wave, - - Bending reflected wave, $\cdots$ Longitudinal transmitted wave, --Longitudinal reflected wave 


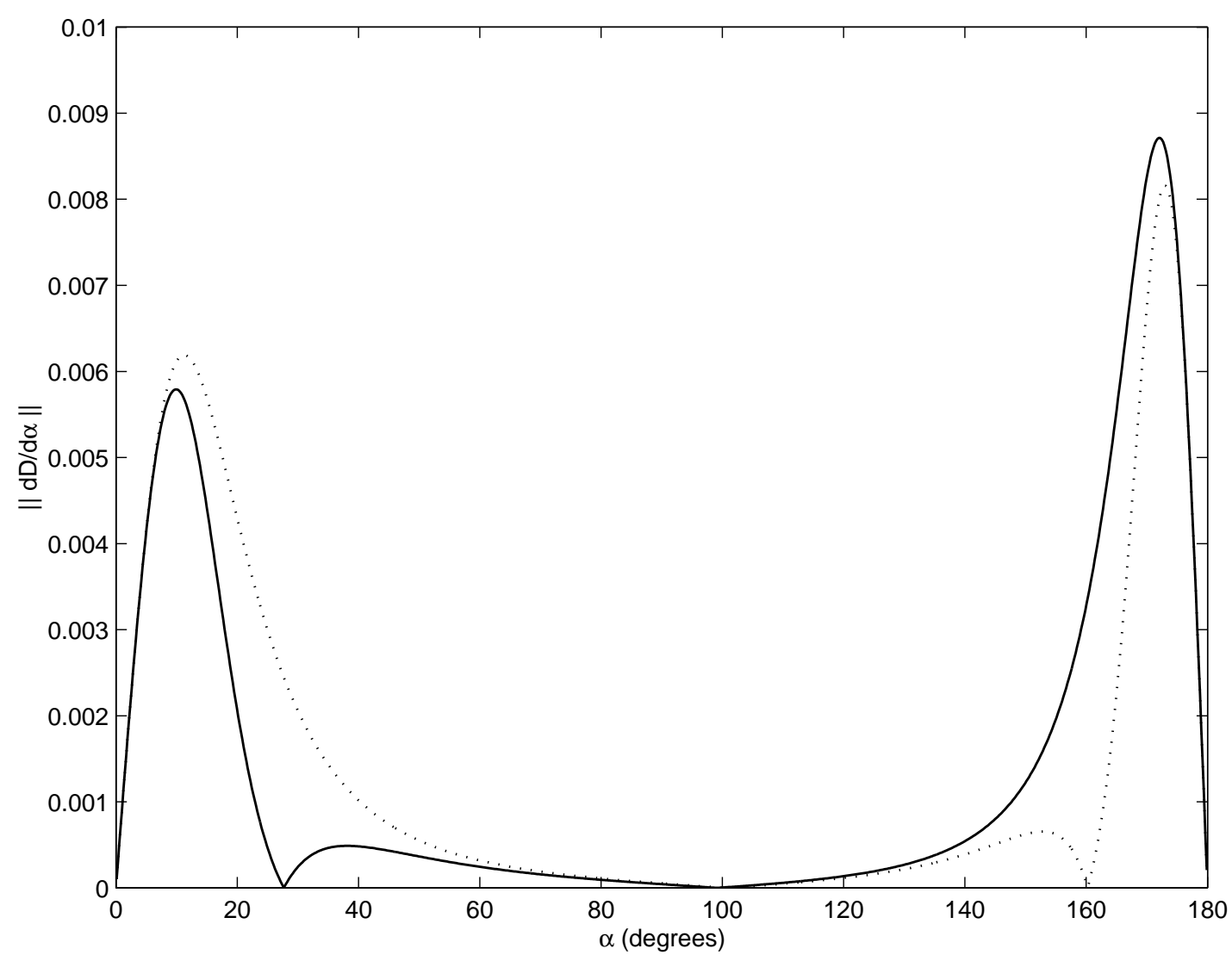

Figure 3: Derivate of modulus of propagative bending waves with respect to coupling angle $\alpha$, versus this angle. - Transmitted wave, $\cdots$ Reflected wave. 


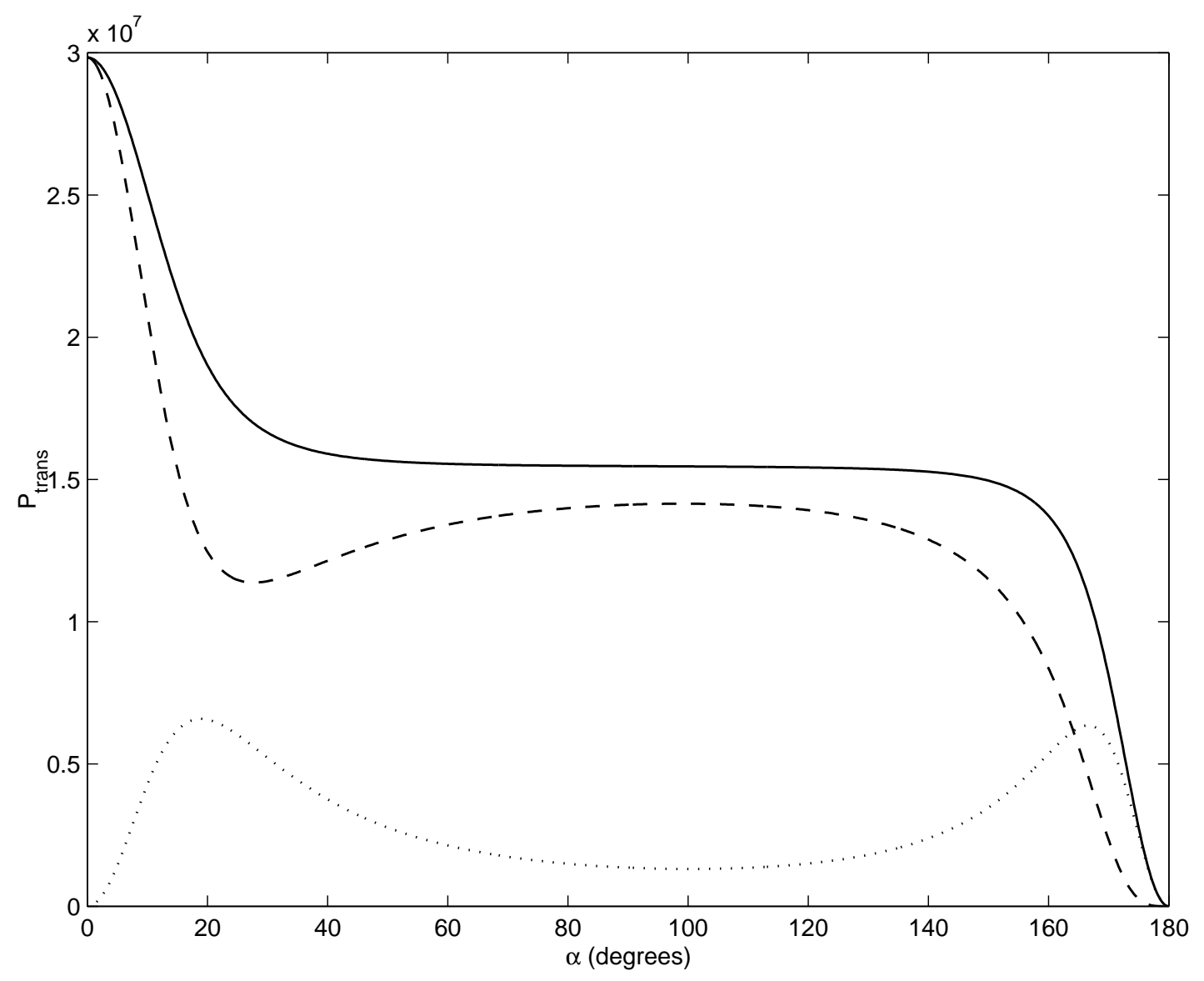

Figure 4: Transmitted powers, versus coupling angle. - Total, - - Bending, - - Inplane 


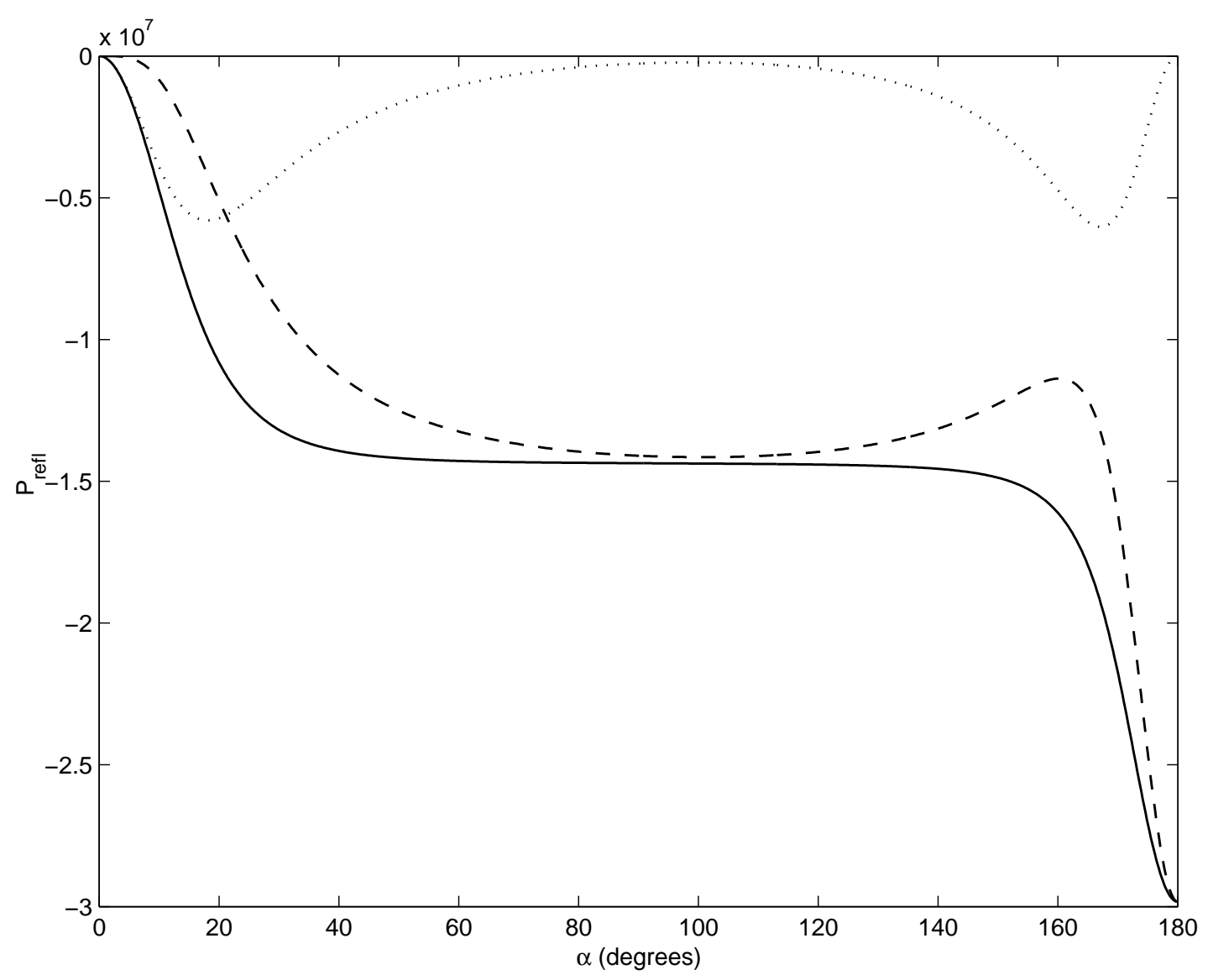

Figure 5: Reflected powers, versus coupling angle. - Total, - - Bending, $\cdots$ Inplane 


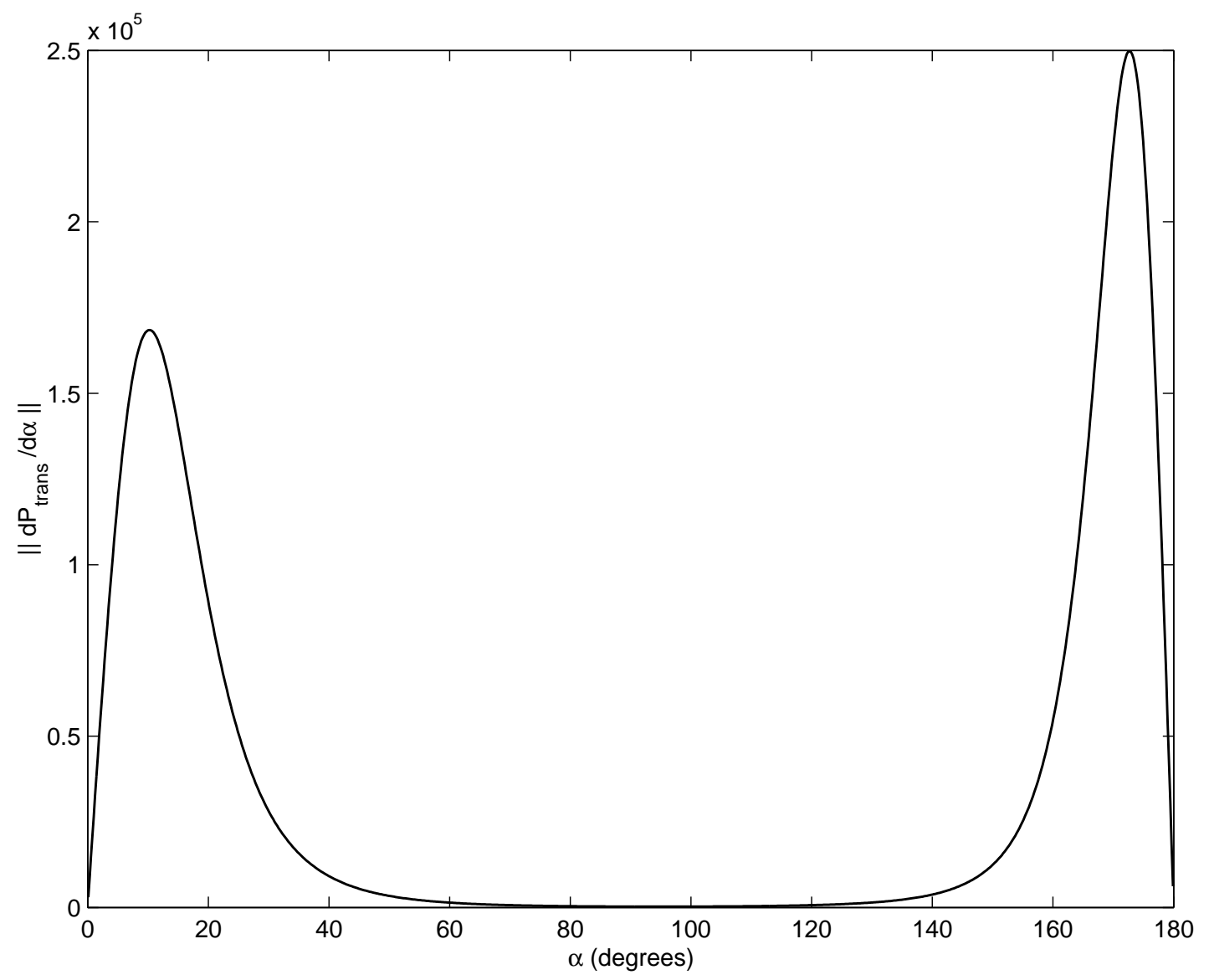

Figure 6: Sensitivity of power versus coupling angle. 


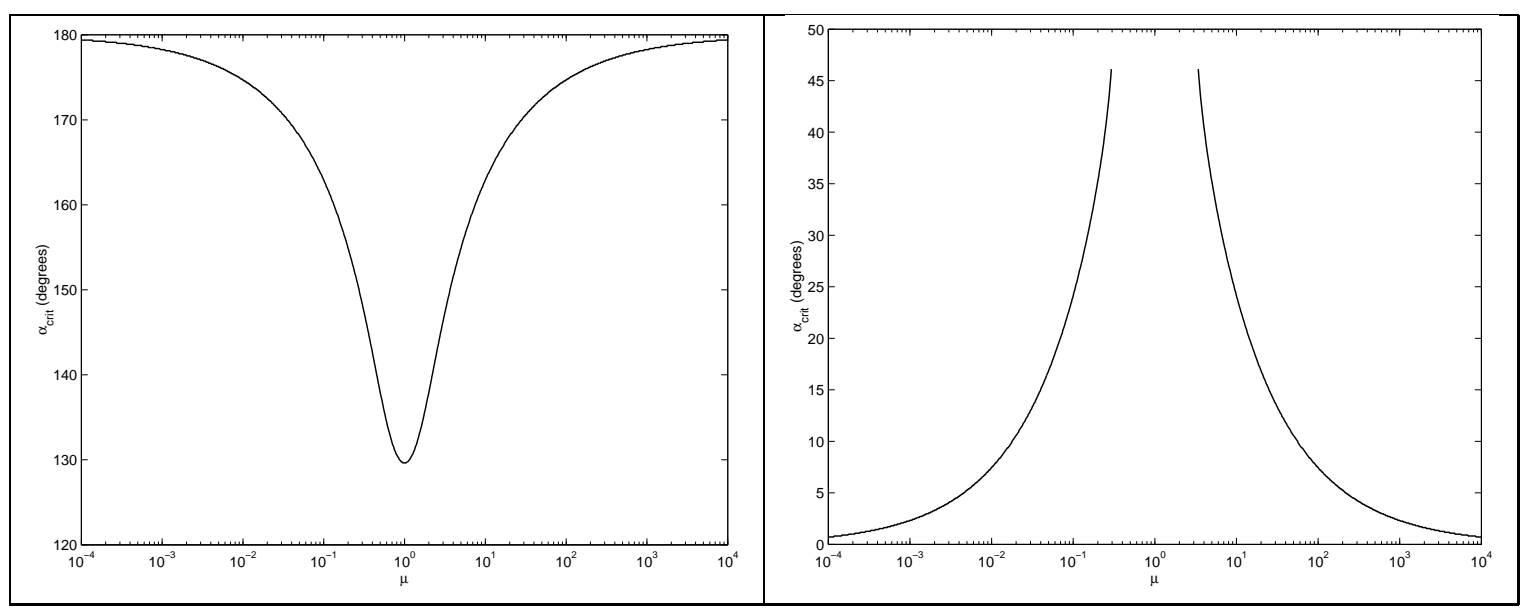

Figure 7: Critical angles (degrees) versus wave numbers ratio $\mu$. 


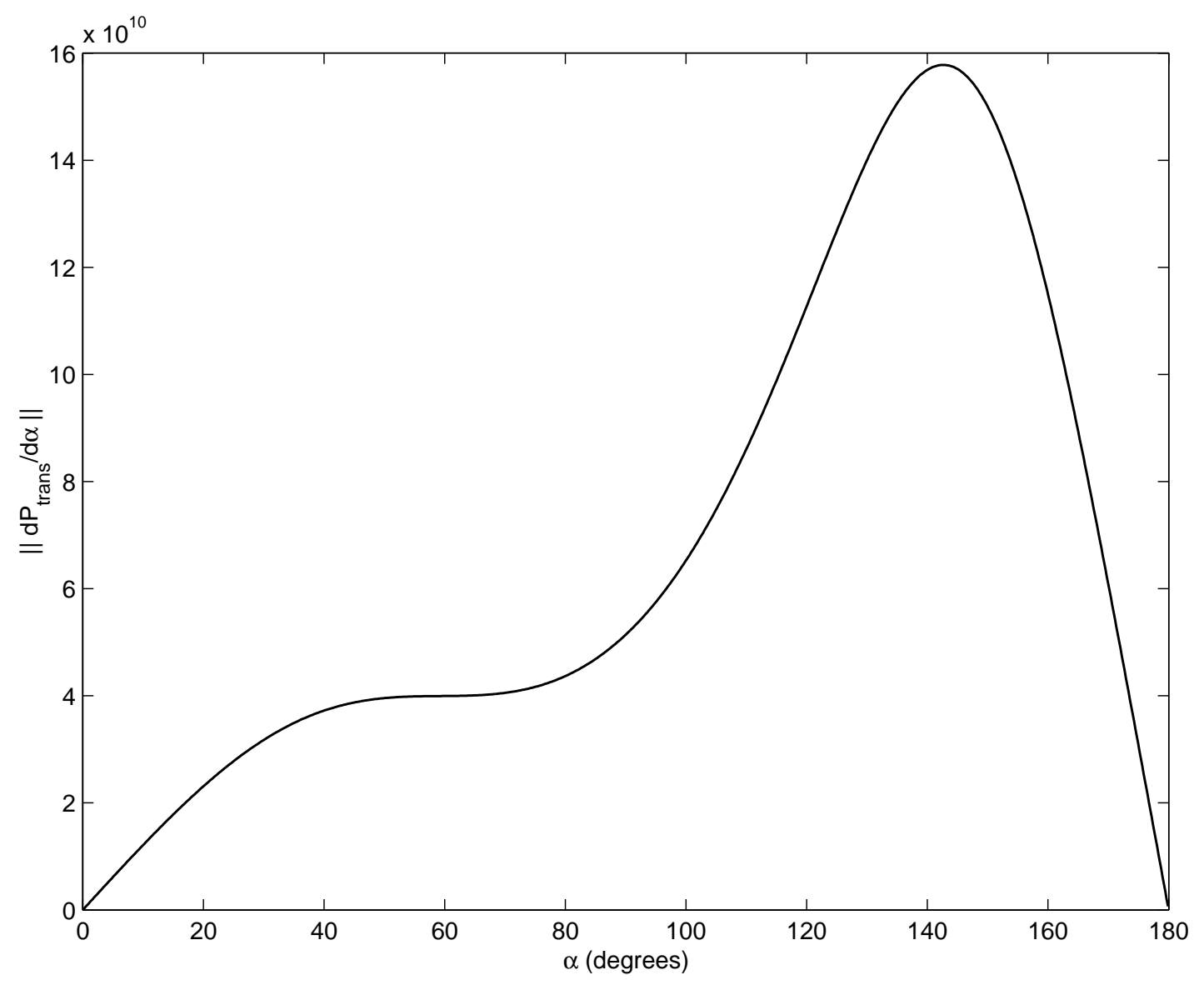

Figure 8: Sensitivity of power versus coupling angle. Limit case. 


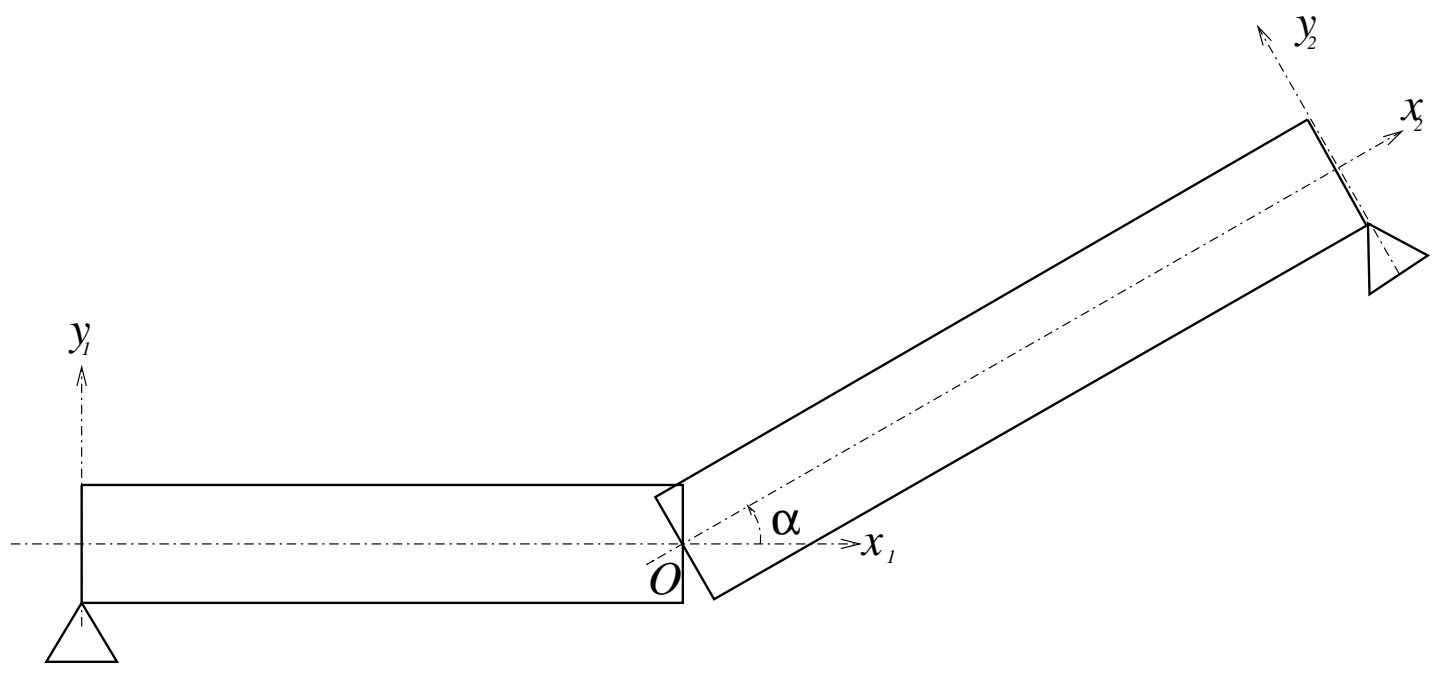

Figure 9: Coupled finite beams 


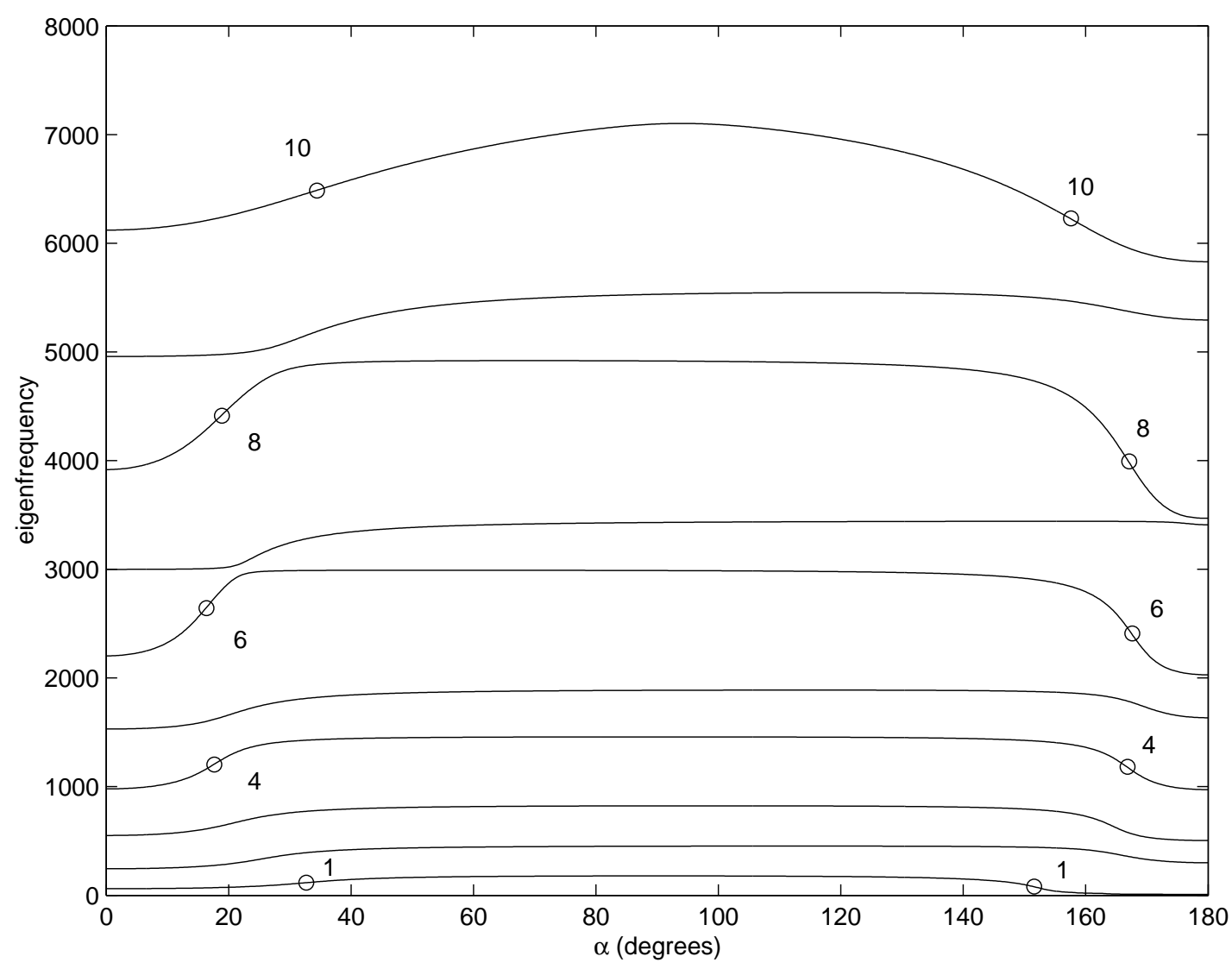

Figure 10: Ten first eigenfrequencies $(\mathrm{Hz})$ versus coupling angle (degrees). Symbols o denote inflexion points of curves. 


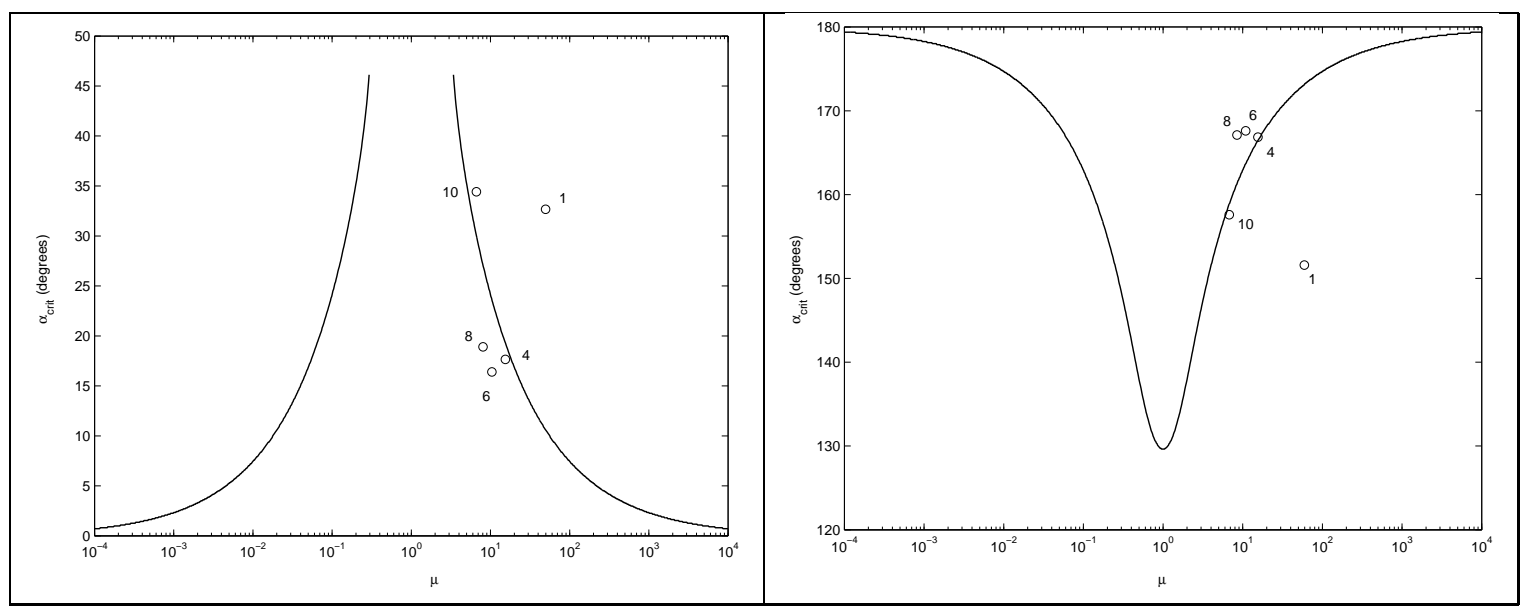

Figure 11: Critical angle versus wave number ratio $\mu$. - coupled semi-infinite beams; o values obtained for modes of finite coupled beam 


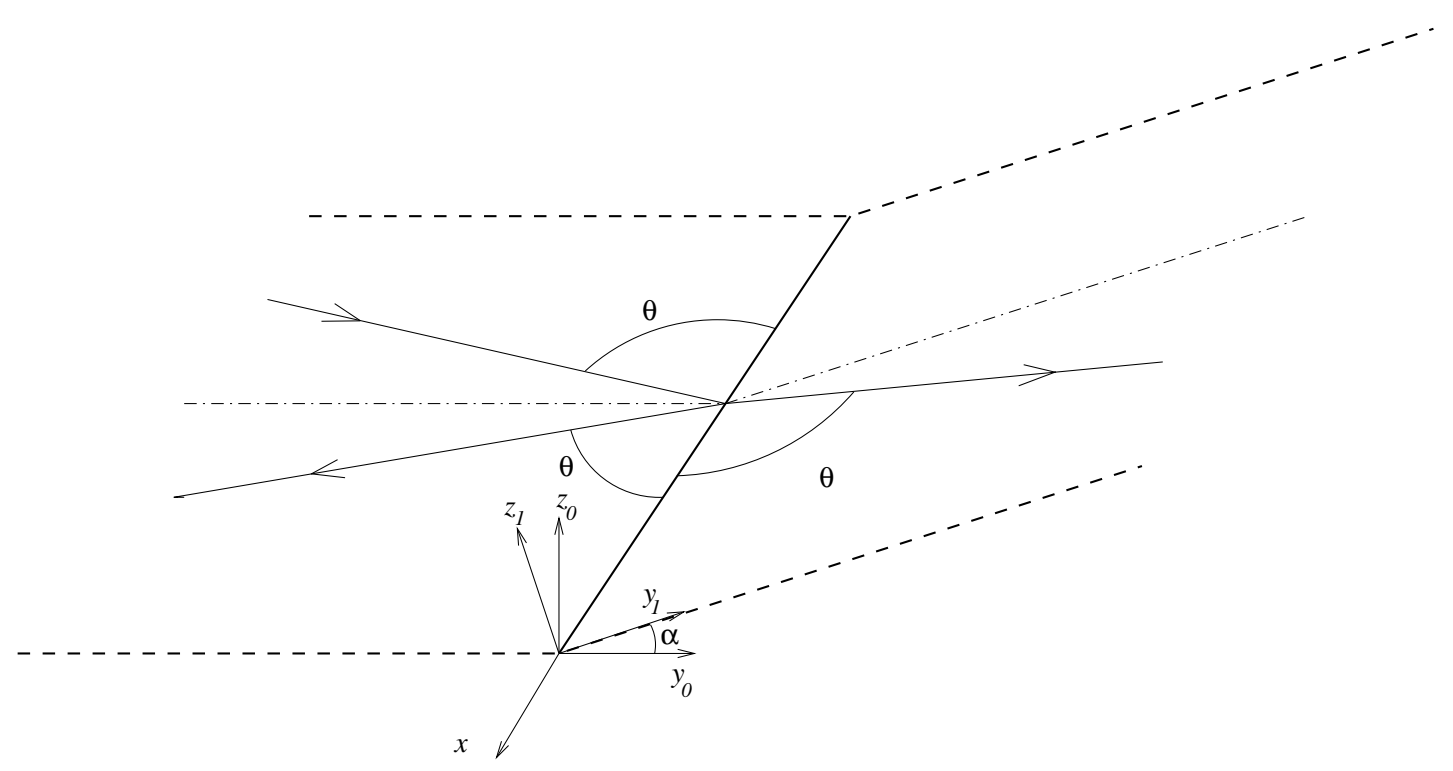

Figure 12: Two semi-infinite coupled plates. 


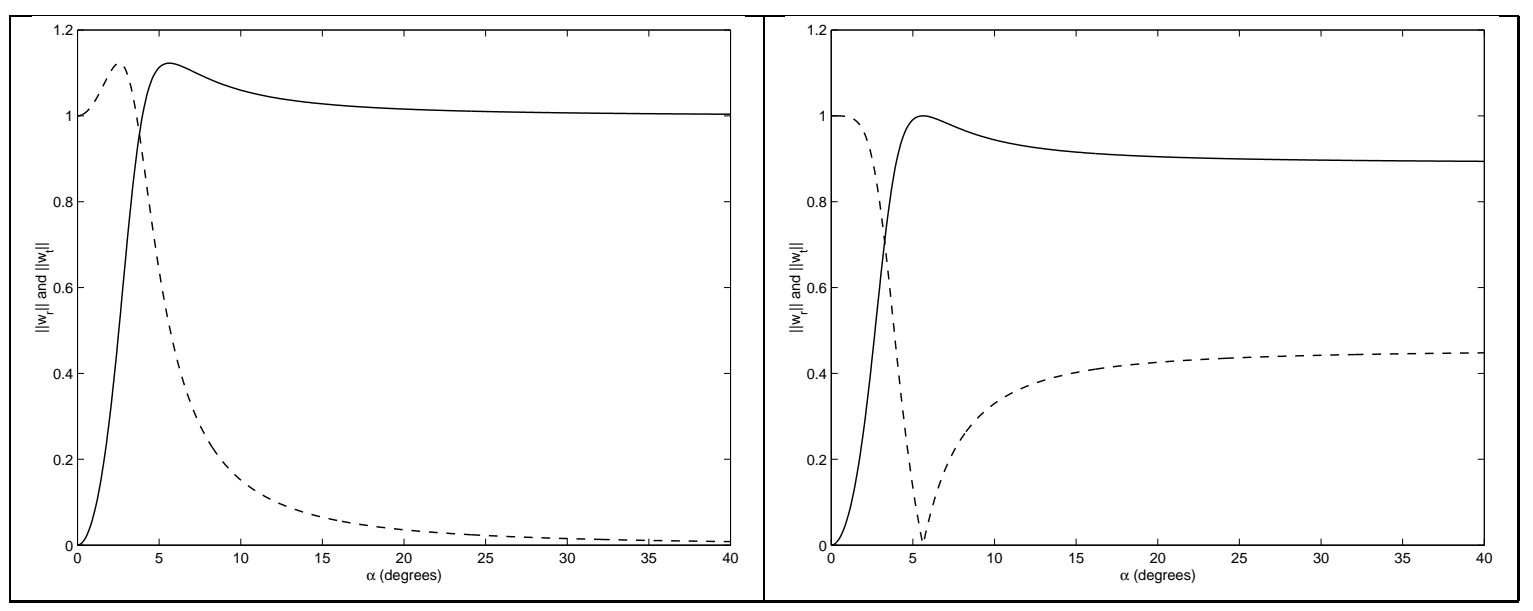

Figure 13: Modulus of bending response, vs. coupling angle. a) response on junction line b) response far from the junction. _ - - transmitted waves; -reflected waves 


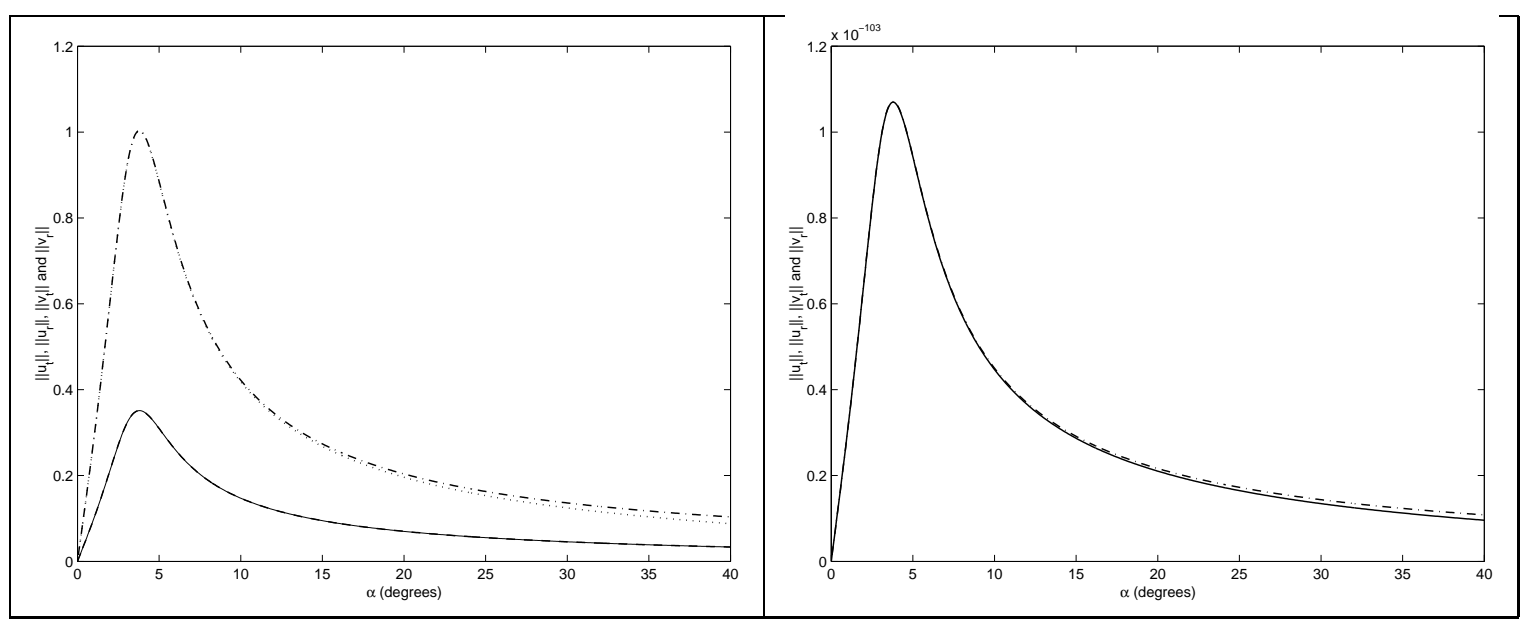

Figure 14: Modulus of in-plane response, vs. coupling angle. a) response on junction line b) response far from the junction - - transmitted $u$ waves; -reflected $u$ waves; -.transmitted $v$ waves; *.reflected $v$ waves 


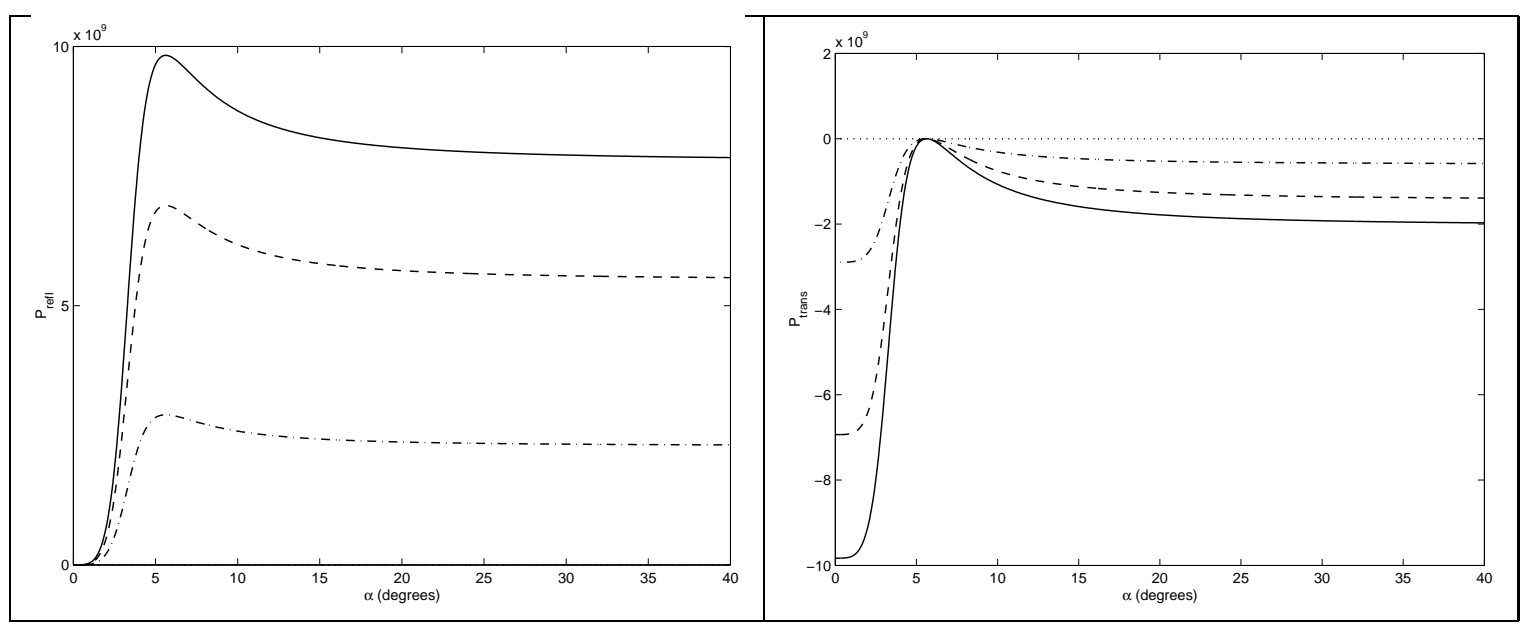

Figure 15: Powers evolution vs. coupling angle. a) reflected powers b) transmitted powers - - Bending moment power; - Shear force power; ·.In-plane power (always zero since these are close field waves); - Total power 


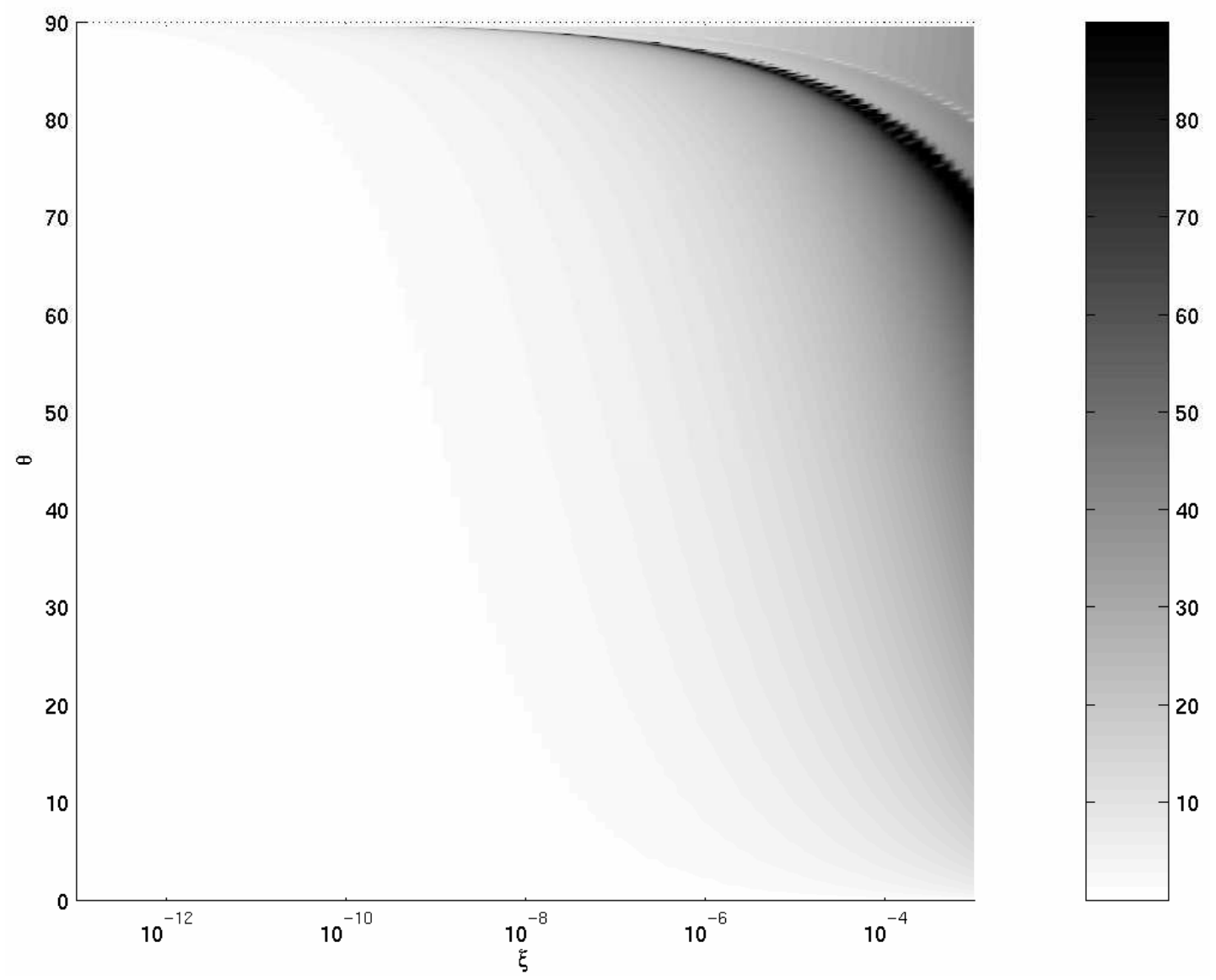

Figure 16: Critical angle (degrees) vs. incident angle $\theta$ and nondimensionnal parameter $\xi . \nu=0.3$ 


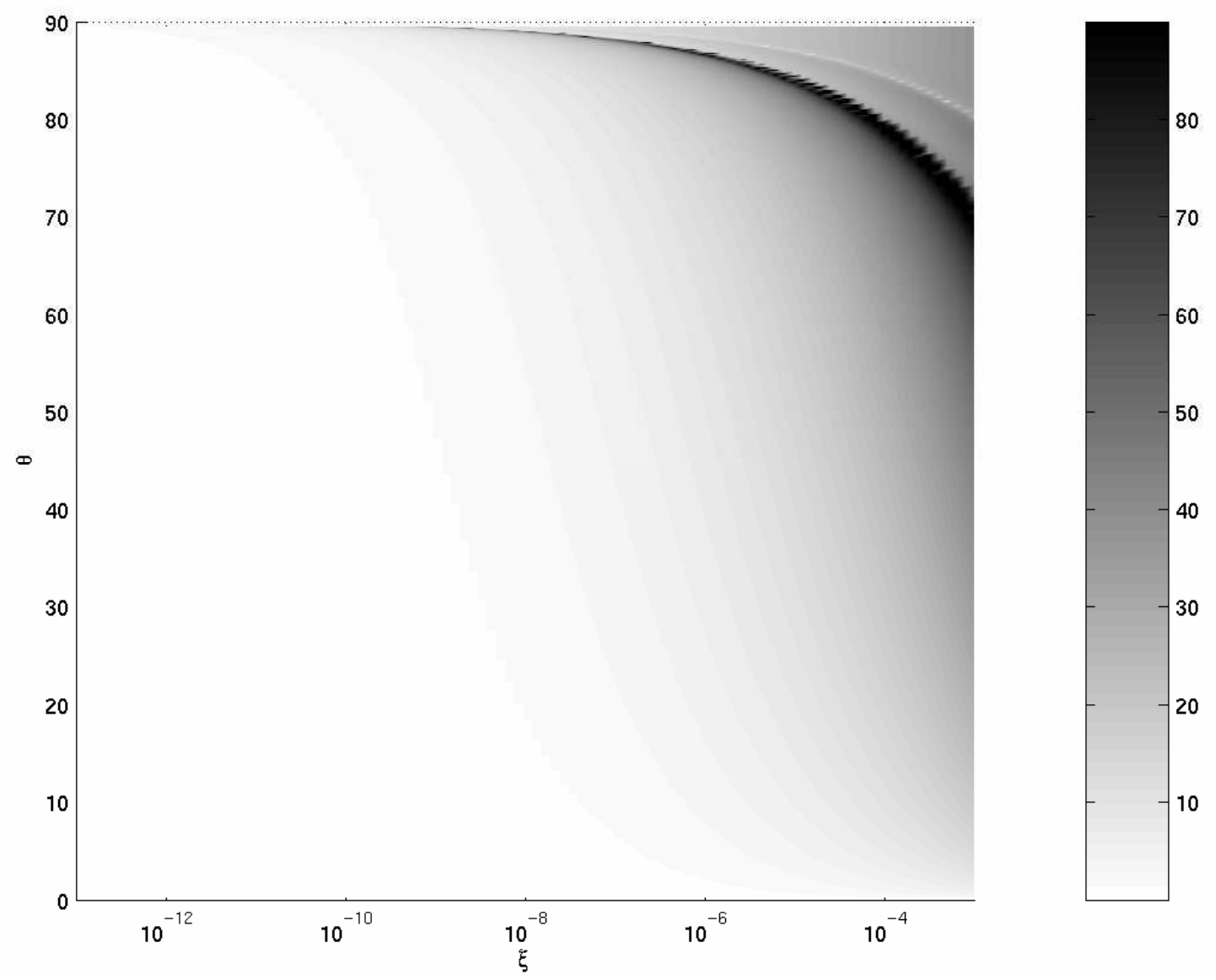

Figure 17: Critical angle (degrees) vs. incident angle $\theta$ and nondimensionnal parameter $\xi . \nu=0.4$ 


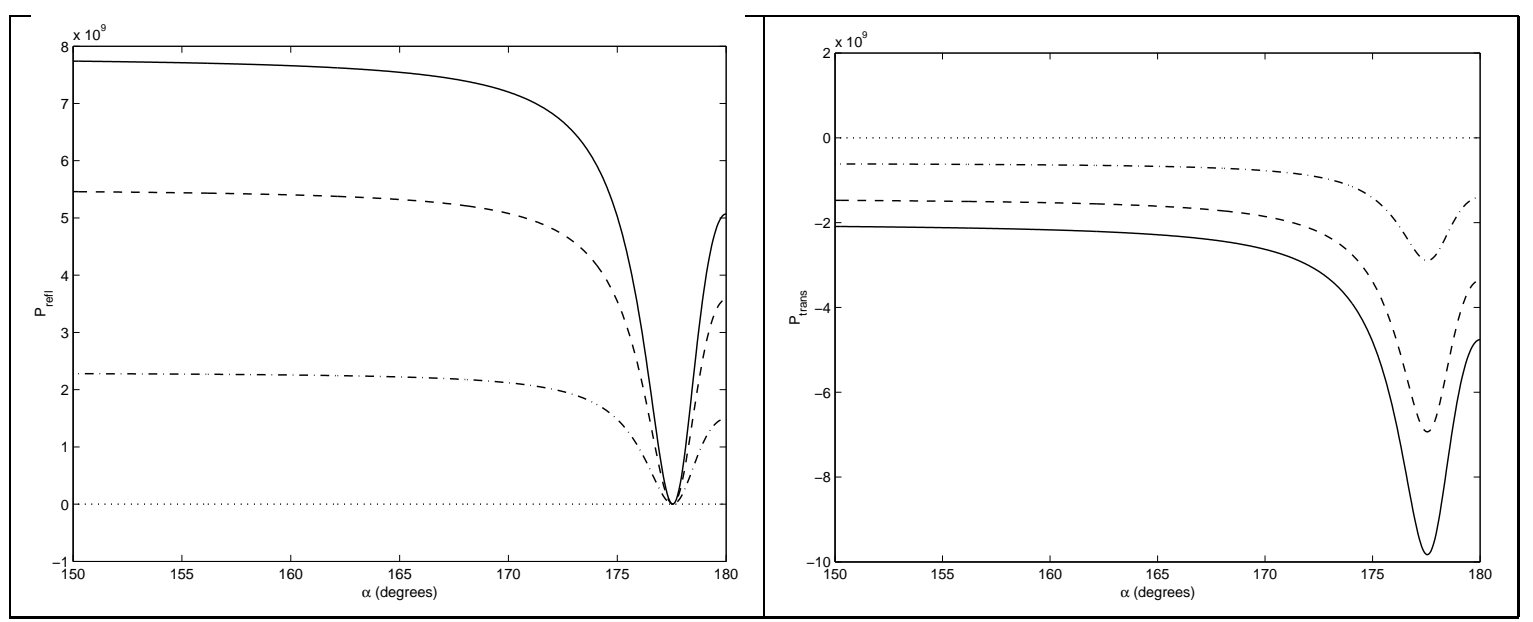

Figure 18: Powers evolution vs. coupling angle. Incident angle $\theta=40^{\circ}$. a) reflected powers b) transmitted powers - - Moment; -.Shear force; ·.In-plane (always zero since these are close field waves); - Total 


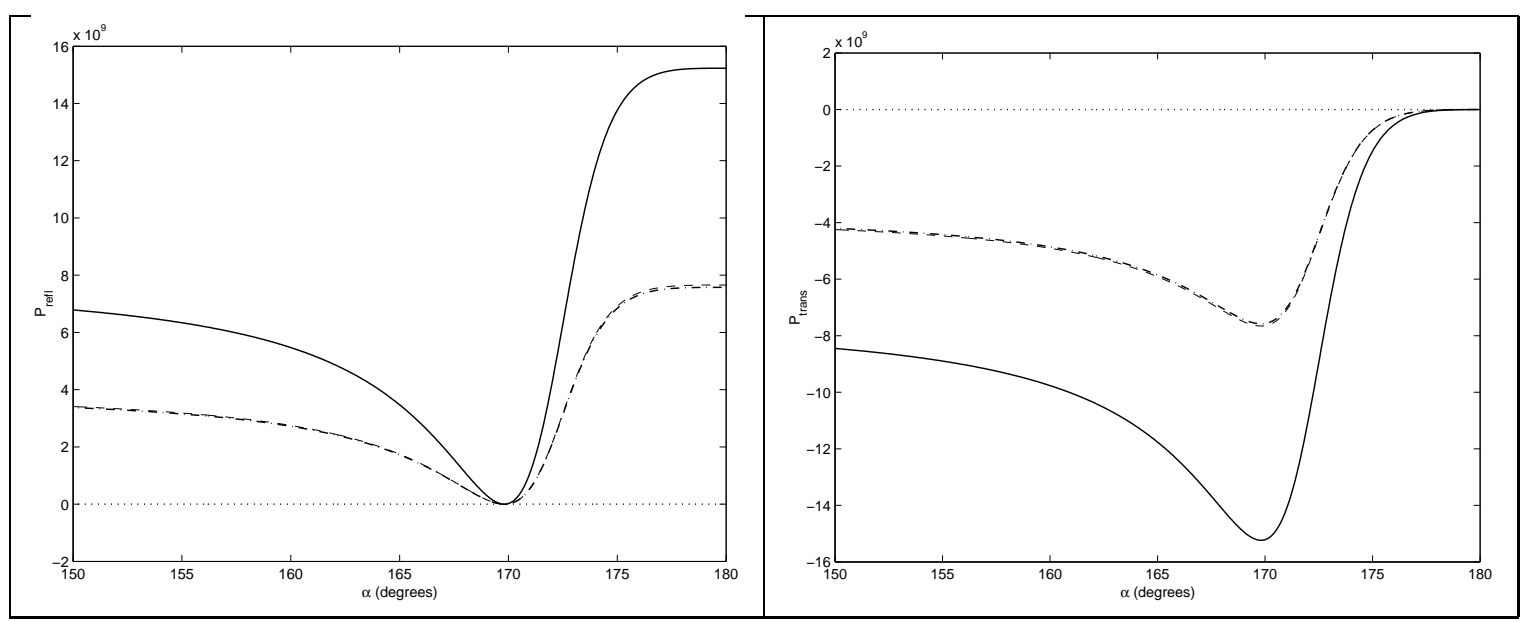

Figure 19: Powers evolution vs. coupling angle. Incident angle $\theta=85^{\circ}$. a) reflected powers b) transmitted powers - - Moment; -.Shear force; ·.In-plane (always zero since these are close field waves); - Total 


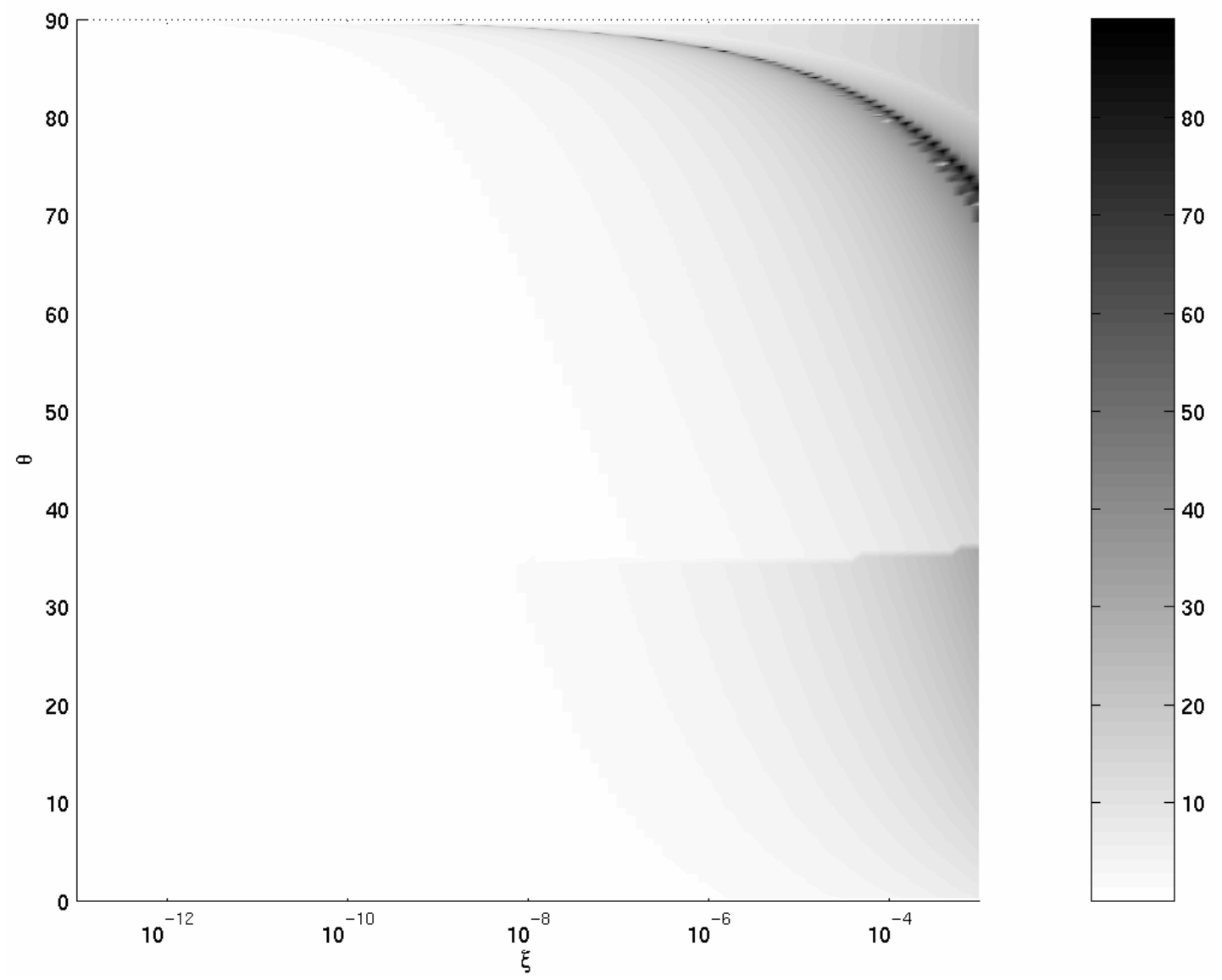

Figure 20: Critical angle (belonging to $90-180$ degrees, plotted value is $180^{\circ}-\alpha_{\text {crit }}$ ) vs. incident angle $\theta$ and nondimensionnal parameter $\xi . \nu=0.3$. 


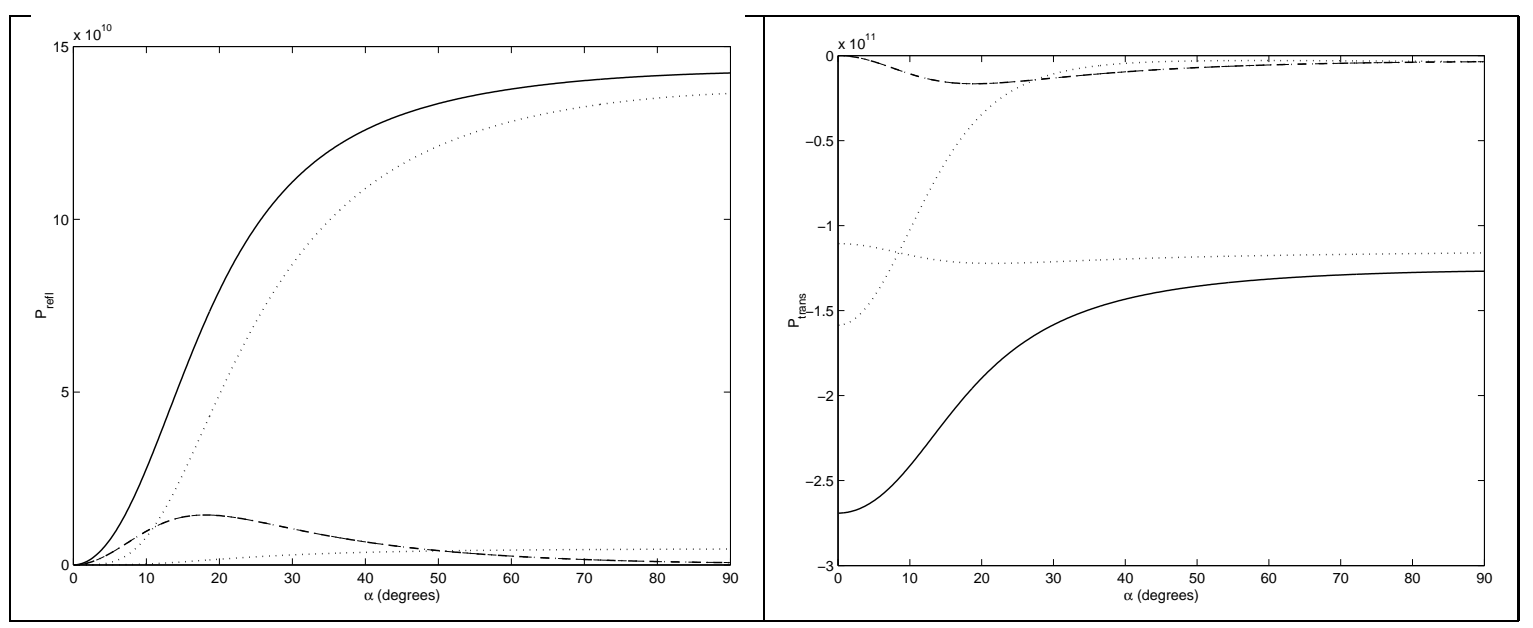

Figure 21: Powers evolution vs. coupling angle. a) reflected powers b) transmitted powers - - Moment; -.Shear force; ·.In-plane; -Total 


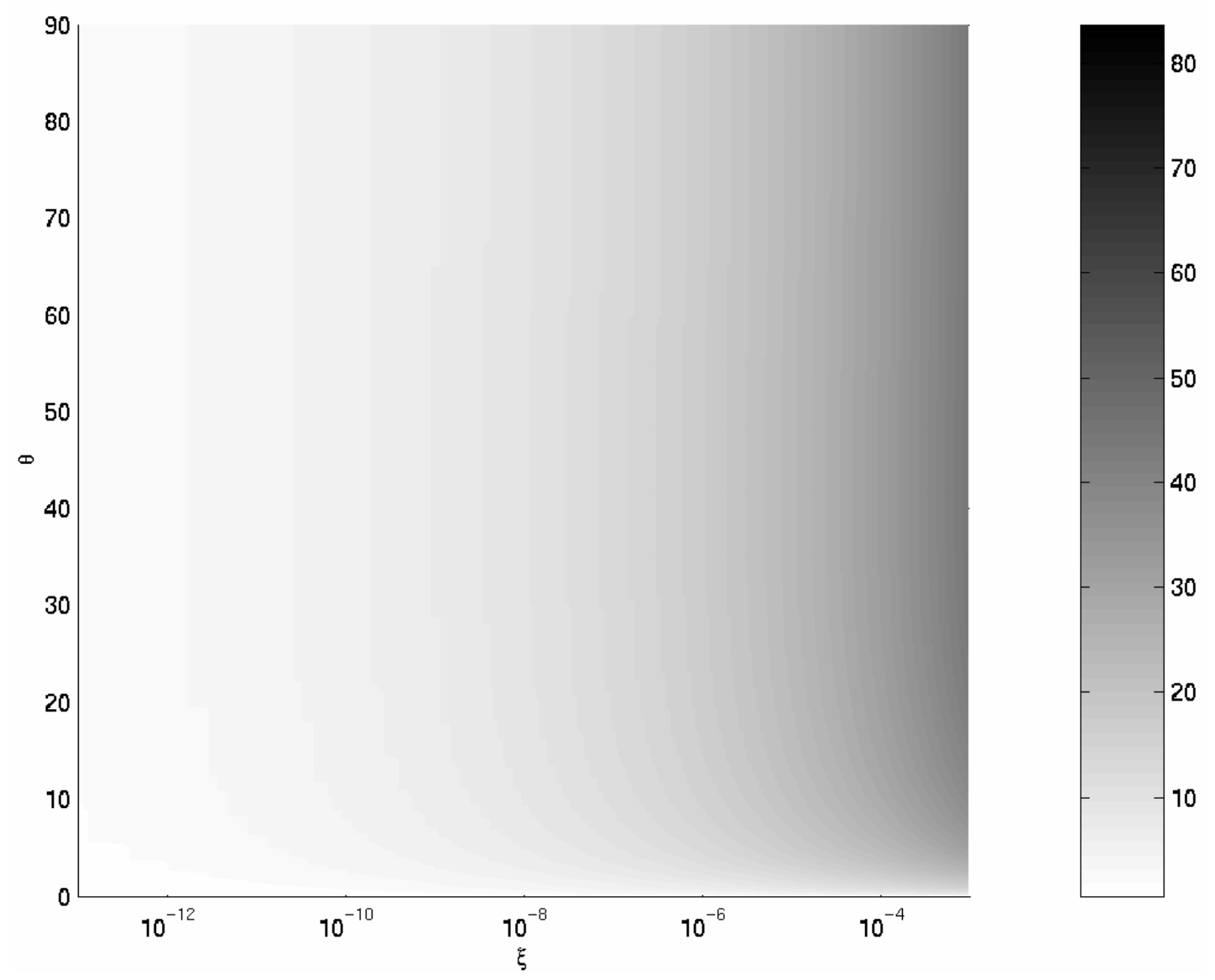

Figure 22: Critical angle (degrees) for incident in-plane longitudinal wave vs. incident angle $\theta$ and nondimensionnal parameter $\xi . \nu=0,3$ 


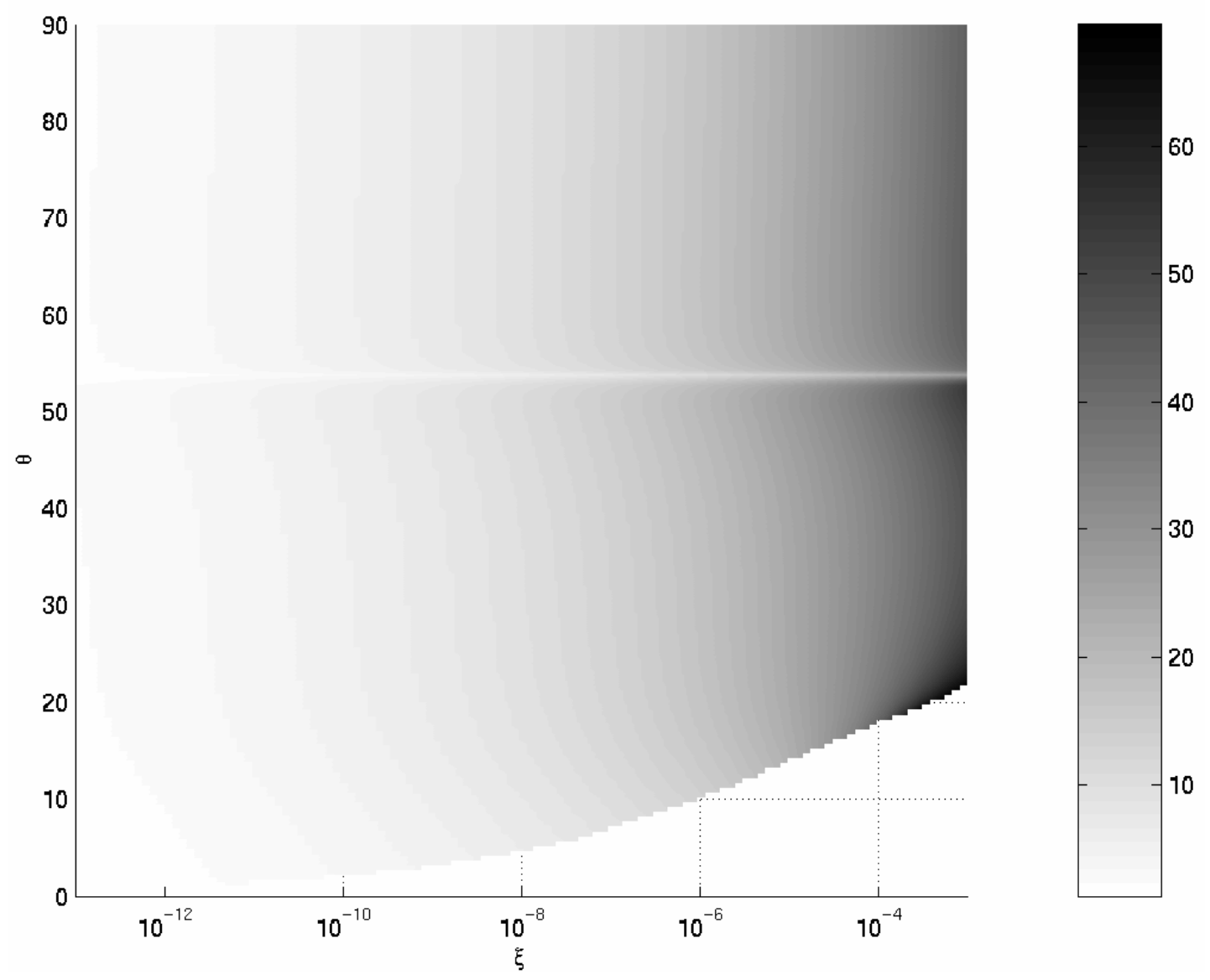

Figure 23: Critical angle (degrees) for incident in-plane shear wave vs. incident angle $\theta$ and nondimensionnal parameter $\xi . \nu=0,3$ 


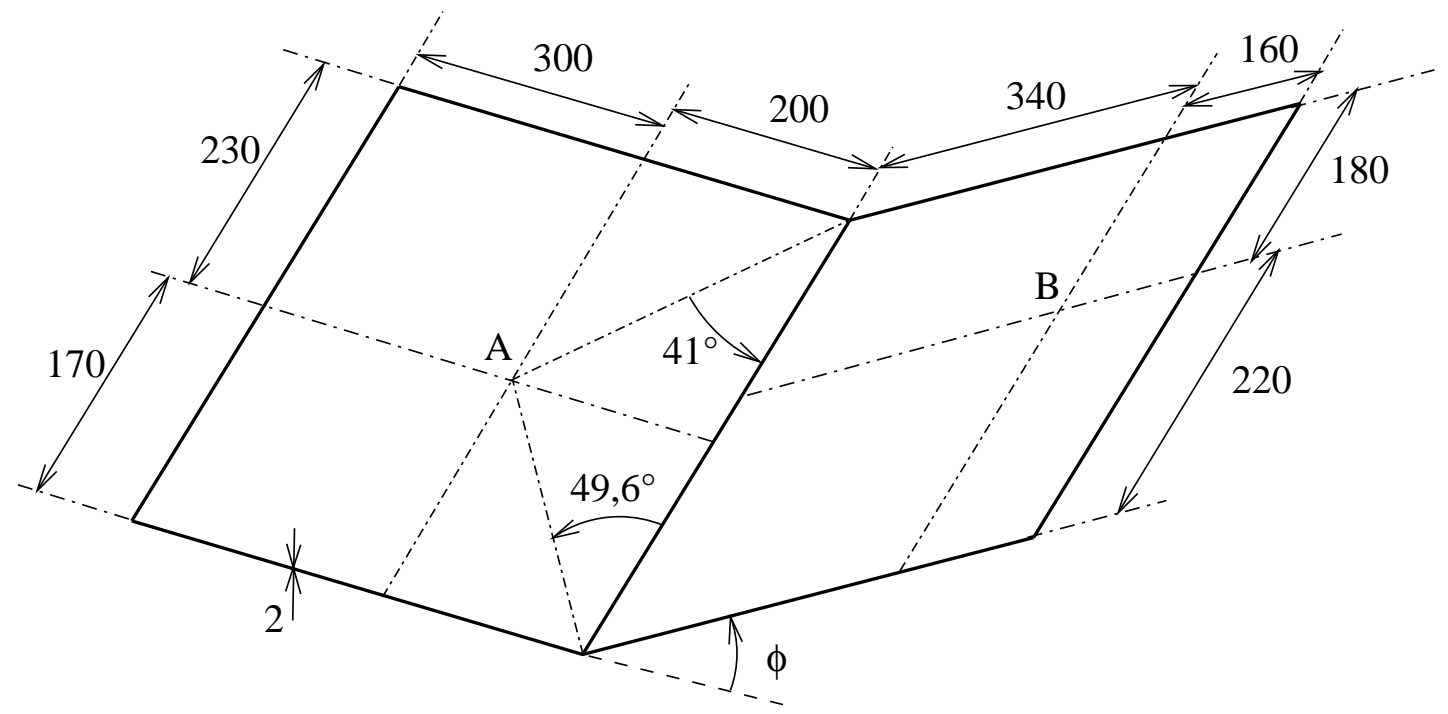

Figure 24: Size (mm) of the coupled finite plates 


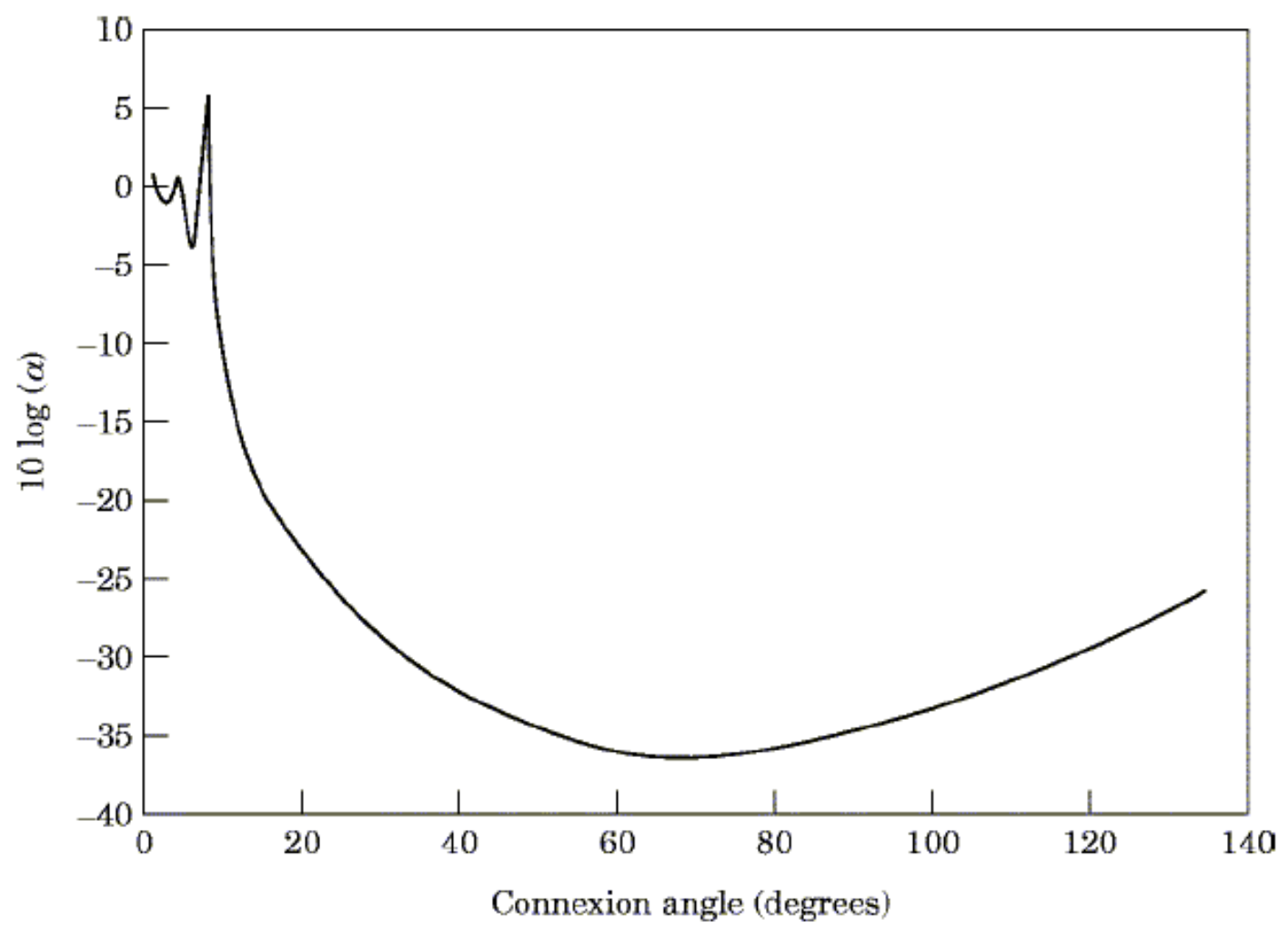

Figure 25: Sensitivity of transfer mobility. After ref [5]. 


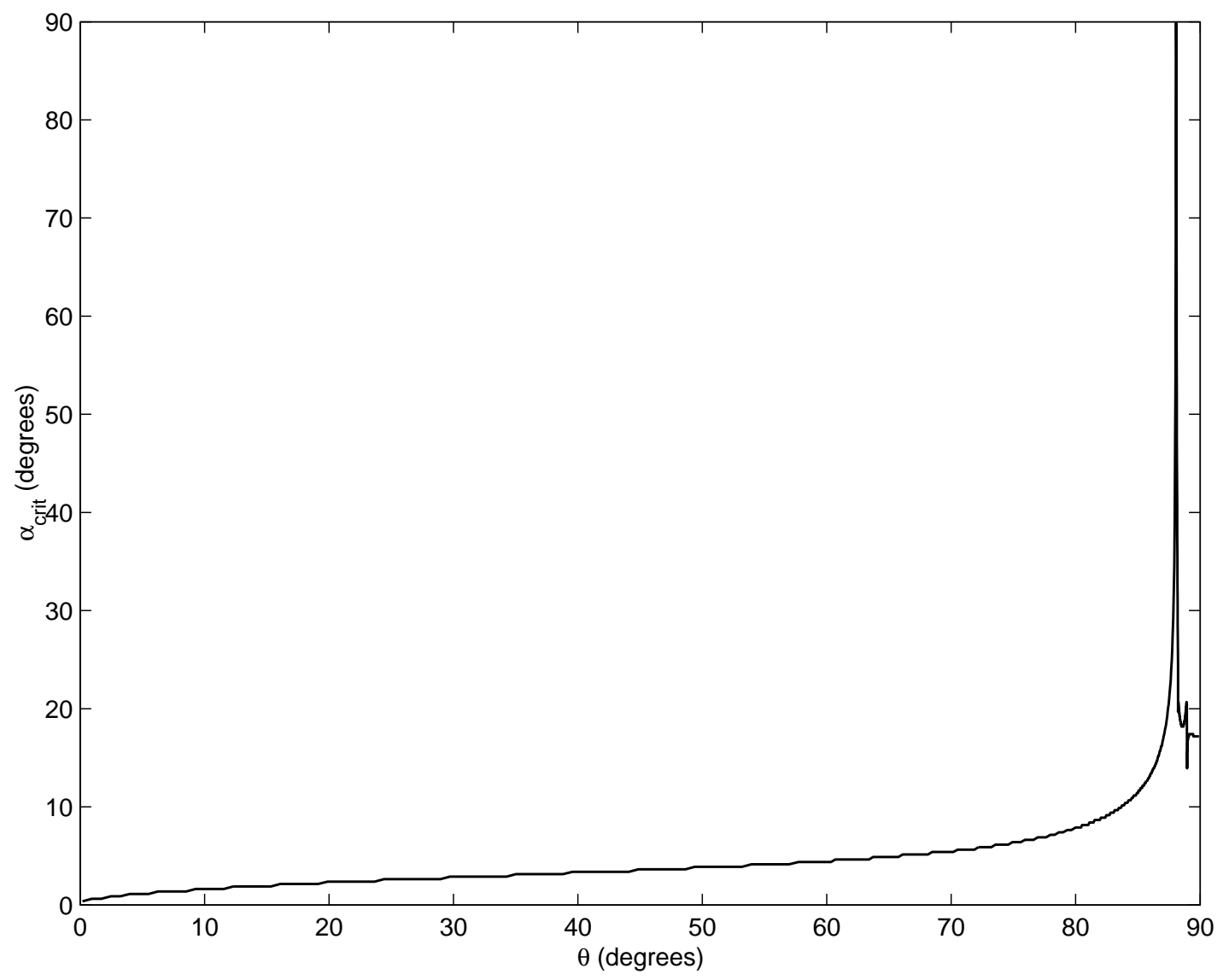

Figure 26: Cut of figure 16, for $\xi=1,23.10^{-7}$ : critical coupling angle versus incidence angle 


\section{List of Figures}

1 Two coupled semi-infinite beams . . . . . . . . . . . . . . . . 49

2 Modulus of amplitudes of propagative waves versus coupling angle.

-Bending transmitted wave, - - Bending reflected wave, $\cdots$ Longitudinal transmitted wave, -. Longitudinal reflected wave . . . . . . . 50

3 Derivate of modulus of propagative bending waves with respect to coupling angle $\alpha$, versus this angle. - Transmitted wave, $\cdots$ Reflected wave. . . . . . . . . . . . . . . . . . 51

4 Transmitted powers, versus coupling angle. - Total, - - Bending, ...In-plane . . . . . . . . . . . . . . . . . 52

5 Reflected powers, versus coupling angle. - Total, - - Bending, ..In-plane . . . . . . . . . . . . . . . . . . 53

6 Sensitivity of power versus coupling angle. . . . . . . . . . . . . 54

$7 \quad$ Critical angles (degrees) versus wave numbers ratio $\mu \ldots \ldots 55$

8 Sensitivity of power versus coupling angle. Limit case. . . . . . . 56

$9 \quad$ Coupled finite beams . . . . . . . . . . . . . . . 57

10 Ten first eigenfrequencies $(\mathrm{Hz})$ versus coupling angle (degrees). Symbols o denote inflexion points of curves. . . . . . . . . . . 58

11 Critical angle versus wave number ratio $\mu$. - coupled semi-infinite beams; o values obtained for modes of finite coupled beam . . . . 59

12 Two semi-infinite coupled plates. . . . . . . . . . . . . 60

13 Modulus of bending response, vs. coupling angle. a) response on junction line b) response far from the junction. - - - transmitted waves; -reflected waves . . . . . . . . . . . . 61 
14 Modulus of in-plane response, vs. coupling angle. a) response on junction line b) response far from the junction - - transmitted $u$ waves; - reflected $u$ waves; -.transmitted $v$ waves; ·.reflected $v$ waves . . . . . . . . . . . . . . . .

15 Powers evolution vs. coupling angle. a) reflected powers b) transmitted powers - - Bending moment power; - Shear force power; ..In-plane power (always zero since these are close field waves); -Total power . . . . . . . . . . . . . . .

16 Critical angle (degrees) vs. incident angle $\theta$ and nondimensionnal parameter $\xi . \nu=0.3 \ldots \ldots \ldots \ldots \ldots$

17 Critical angle (degrees) vs. incident angle $\theta$ and nondimensionnal parameter $\xi . \nu=0.4 \ldots \ldots \ldots \ldots$

18 Powers evolution vs. coupling angle. Incident angle $\theta=40^{\circ}$. a) reflected powers b) transmitted powers _ - Moment; -.Shear force; ·.In-plane (always zero since these are close field waves);

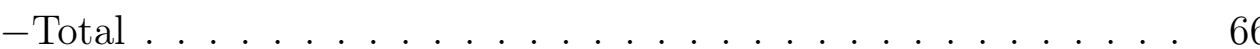

19 Powers evolution vs. coupling angle. Incident angle $\theta=85^{\circ}$. a) reflected powers b) transmitted powers _ - Moment; -.Shear force; ..In-plane (always zero since these are close field waves); -Total . . . . . . . . . . . . . .

20 Critical angle (belonging to 90-180 degrees, plotted value is $180^{\circ}-$ $\left.\alpha_{c r i t}\right)$ vs. incident angle $\theta$ and nondimensionnal parameter $\xi . \nu=$ 0.3 .

21 Powers evolution vs. coupling angle. a) reflected powers b) transmitted powers $\quad$ - - Moment; -.Shear force; ·.In-plane; -Total . 
22 Critical angle (degrees) for incident in-plane longitudinal wave vs. incident angle $\theta$ and nondimensionnal parameter $\xi . \nu=0,3 \ldots 70$

23 Critical angle (degrees) for incident in-plane shear wave vs. incident angle $\theta$ and nondimensionnal parameter $\xi . \nu=0,3 \ldots 71$

24 Size $(\mathrm{mm})$ of the coupled finite plates . . . . . . . . . . . 72

25 Sensitivity of transfer mobility. After ref [5]. . . . . . . . . . 73

26 Cut of figure 16, for $\xi=1,23.10^{-7}$ : critical coupling angle versus incidence angle . . . . . . . . . . . . . . . . . . 74 